\title{
Nulla executio sine titulo: el escrutinio de los laudos en la práctica del arbitraje internacional como mecanismo para optimizar la ejecución de los laudos en el Perú Nulla executio sine titulo: the scrutiny of awards in the practice of international arbitration as a mechanism to optimize the enforcement of awards in Peru
}

\author{
JOSÉ DOMINGO RIVAROLA REISZ" \\ Payet, Rey, Cauvi, Pérez Abogados
}

\section{FABIO NÚÑEZ DEL PRADO CHAVES** Universidad Carlos III de Madrid}

Resumen: ¿Qué es lo «liquidable» y cómo debe ser este concepto enfrentado por los árbitros y jueces? ¿Puede un juez en ejecución determinar conceptos como costas, intereses y/o las penalidades sin que el tribunal arbitral le haya brindado ningún parámetro? ¿Qué derechos fundamentales están en juego? En función de la práctica arbitral internacional, nos ocuparemos de los problemas que surgen por la división de funciones entre jueces y árbitros en relación con la ejecución de lo «liquidable».

Palabras clave: autonomía privada, arbitraje internacional, Cámara de Comercio Internacional, efectividad, ejecución, escrutinio, laudo, reglamento arbitral

Abstract: What is «liquidable» and how should this concept be faced by arbitrators and judges? Can an enforcement judge determine concepts such as costs, interests and/or penalties if the arbitral tribunal has not provided any parameters? What fundamental rights might be violated? In accordance with international arbitration practice, we will deal here with the problems arising from the division of functions between judges and arbitrators in relation to the enforcement of the «liquidable» concepts.

Key words: party autonomy, international arbitration, International Chamber of Commerce, effectiveness, enforcement, scrutiny, award, arbitral rules

* Socio del Estudio Payet, Rey, Cauvi, Pérez en el área de litigio arbitral y judicial. Profesor ordinario de la Pontificia Universidad Católica del Perú en el área de solución de controversias, arbitraje y prueba. Código ORCID: 0000-0002-4557-3027. Correo electrónico: drr@ prc.com.pe

** Asociado internacional del área de arbitraje de Uría Menéndez. Profesor de la Universidad Carlos III de Madrid, Pontificia Universidad Católica del Perú, Universidad Peruana de Ciencias Aplicadas y la Universidad ESAN. Miembro de la asociación internacional Arbitration Academy. Ex miembro del Consejo Editorial de Derecho PUCP.

Códico ORCID: 0000-0001-6614-6561. Correo electrónico: fabio.np05@gmail.com 
IV. LA APLICACIÓN INMEDIATA DEL ARTÍCULO 139 DE LA CONSTITUCIÓN AL ARBITRAJE.- V. LA EJECUCIÓN DEL LAUDO Y SUS TROPIEZOS.- VI. LOS PROBLEMAS DE LA EJECUCIÓN DEL LAUDO EN LA JURISPRUDENCIA.VII. CONFLICTO DE PRINCIPIOS CONSTITUCIONALES.- VIII. ¿QUÉ ES EL ESCRUTINIO DE LOS LAUDOS?- IX. EL ESCRUTINIO DE LOS LAUDOS COMO MECANISMO QUE MAXIMIZA LA AUTONOMÍA PRIVADA Y LA EFECTIVIDAD DE LAS DECISIONES JURISDICCIONALES.- X. UNA PERSPECTIVA PRÁCTICA: ¿POR QUÉ EL ESCRUTINIO DE LOS LAUDOS ES NECESARIO?- XI. LAS CRÍTICAS INFUNDADAS ALESCRUTINIO DELOSLAUDOS.-XII.ELÉXITO DELESCRUTINIO DE LOS LAUDOS A NIVEL INTERNACIONAL.- XIII. CONCLUSIONES.

\section{INTRODUCCIÓN}

Es nula la ejecución judicial sin título que la sustente. Se ha escrito ya sobre este aforismo latino (nulla executio sine titulo) y sobre los problemas de la codificación peruana (Ariano, 2010). Lo cierto es que el proceso judicial ejecutivo, de ejecución o «único de ejecución» (Código Procesal Civil, artículos 688ss.) supone vías procedimentales céleres, cuyo objetivo final es optimizar los derechos de los acreedores, limitando las posibilidades de contradicción de los deudores ${ }^{1}$. En los procesos para la ejecución de títulos, la principal comprobación que debe hacer el juzgador es si existe y está vigente el título que sustenta la ejecución y si este está libre de defectos.

En el caso extremo, cuando existe una sentencia judicial de condena al pago de dinero, las únicas defensas del deudor son las causales de extinción de las obligaciones reguladas en el Libro V del Código Civil. Sin embargo, en cualquier caso, un presupuesto para la ejecución es que la obligación que el título representa, cuando esta sea dineraria, sea líquida o liquidable. En caso contrario, la ejecución no es procedente, según se deriva de la regulación del Código Procesal Civil. Un laudo arbitral es un título jurisdiccional susceptible de ejecución. Aunque tiene una vía procedimental diferenciada para la ejecución (Ley de Arbitraje, artículo 68), la esencia es la misma: el laudo debe contener una obligación líquida o liquidable. ¿Qué es lo «liquidable» y cómo debe ser este concepto enfrentado por los árbitros y jueces? A partir de las reglas previstas en la vigente Ley de Arbitraje y en función de la práctica arbitral internacional, nos ocuparemos en este artículo de los problemas que surgen por la división de funciones entre jueces y árbitros en relación con la ejecución de lo «liquidable».

Dicha división de funciones surge cuando las partes de un contrato deciden incorporar un convenio arbitral que someta una controversia

1 No en vano señala Ariano que la «efectividad de una ejecución (cualquier ejecución) debe partir de un claro "título" para proceder a ella, pues mientras más "certero" será este será menor la posibilidad de una oposición (válida) a que la misma se lleve a cabo» (Ariano, 2010, p. 93). 
a la jurisdicción arbitral. Con ello buscan evitar los formalismos y rigideces propios de un proceso judicial, con el fin de tener amplia y flexible posibilidad de prueba y alegación, mayor oralidad y mayor celeridad. Así, las partes normalmente pactan un arbitraje para diseñar un procedimiento arbitral acorde con sus necesidades. Pensemos, por ejemplo, en la necesidad de contar con un árbitro especializado en la cuestión controvertida y con tiempo para dedicarse a la tarea. Controversias relativas a complejos contratos de obra entre privados o a contratos de concesión entre el Estado y empresas para la construcción, operación y mantenimiento de puertos, aeropuertos o carreteras requieren especialización y dedicación casi exclusiva al arbitraje.

Hay necesidades más obvias y básicas que la celeridad, la especialización o la flexibilidad. La más obvia necesidad de las partes es que la decisión del tribunal arbitral cobre vida transformando un estado de cosas y proyectándose en la realidad de los hechos de la manera más rápida y efectiva posible (Salcedo, 2008, p. 202). En otras palabras, las partes no esperan solamente obtener un laudo favorable, sino que buscarán que este se ejecute hasta sus últimas consecuencias (p. 201).

Las partes buscan, en buena cuenta, que los mandatos y las consideraciones del tribunal arbitral no queden solo «en el papel» como declaraciones de buenas intenciones, al punto que se habla del derecho a la efectividad de las decisiones jurisdiccionales. Ello incluye al arbitraje, como ha señalado con acierto nuestro Tribunal Constitucional al entender que el derecho a la tutela procesal efectiva es exigible respecto de los laudos arbitrales (Fernando Cantuarias Salaverry c. Corte Superior de Justicia de Lima, fundamentos 17 y 18).

La efectividad del laudo arbitral es una necesidad básica que las partes pretenden satisfacer cuando acuerdan arbitrar una controversia mediante un convenio arbitral. Habrá efectividad cuando el laudo se vea materializado en la realidad (Priori, 2009, p. 80). Y, para ese propósito, se requiere de una correcta actuación de los jueces y árbitros. Los árbitros no tienen la facultad de convocar a la fuerza pública para transformar la realidad (Ley de Arbitraje, artículo 67, inc. 2) y que el laudo se ejecute hasta sus últimas consecuencias. Asimismo, los jueces no pueden convocar a la fuerza pública si el mandato contenido en el laudo es ambiguo, incierto o indeterminado, pues se requiere que la obligación esté determinada.

Para el caso de las obligaciones dinerarias, el que la obligación esté determinada significa que la obligación sea, obviamente, líquida. Sin embargo, lo que comúnmente causa confusión es el concepto de lo liquidable, como hemos ya anticipado. ¿Cómo se ejecuta un laudo con obligación liquidable? ¿Qué es lo liquidable y cómo asegurarse de que el tribunal arbitral satisfaga la necesidad de las partes de tener un

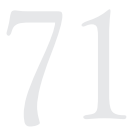

NULLA EXECUTIO SINE TITULO: EL ESCRUTINIO DE LOS LAUDOS EN LA PRÁCTICA DEL ARBITRAJE INTERNACIONAL COMO

MECANISMO

PARA OPTIMIZAR LA EJECUCIÓN DE LOS LAUDOS EN EL PERÚ

NULLA EXECUTIO SINE TITULO:THE SCRUTINY OF AWARDS IN THE PRACTICE OF INTERNATIONAL ARBITRATION AS A MECHANISM TO OPTIMIZE THE ENFORCEMENT OF AWARDS IN PERU 
laudo liquidable y, por lo tanto, efectivo? ¿Qué ocurre si los parámetros del laudo no son suficientes para que la jurisdicción ordinaria practique la liquidación del monto adeudado?

Consideramos que los jueces y árbitros, al responder estas preguntas, deben tener en mente el derecho a la efectividad de las decisiones jurisdiccionales como norte de su labor. El proceso debe ser visto desde fuera de él para ver como él sirve a algo distinto a sí mismo (Priori, 2003, p. 274). El proceso está al servicio del derecho material y no al revés.

\section{LA TUTELA JURISDICCIONAL EFECTIVA Y EL DERECHO A LA EFECTIVIDAD DE LAS DECISIONES JURISDICCIONALES}

Lo trascendente en un proceso arbitral o judicial es tutelar de manera efectiva las situaciones jurídicas materiales. El énfasis está en el resultado, antes que en el medio. Desde un punto de vista constitucional, el sistema procesal que sea diseñado por el legislador debe tratar de asegurar que el proceso cumpla el fin para el que está previsto. Dicho fin es, entre otros, satisfacer la necesidad de las partes o justiciables de tener una decisión definitiva y que transforme la realidad de manera coercitiva ante la falta de cumplimiento espontáneo (Chamorro, 1994, p. 276)².

Al respecto, el artículo 139, inciso 3 de la Constitución Política del Perú de 1993 dispone lo siguiente:

Artículo 139.- Son principios y derechos de la función jurisdiccional: [...]

3.- La observancia del debido proceso y la tutela jurisdiccional.

El derecho a la tutela jurisdiccional efectiva es un derecho de contenido complejo, puesto que está conformado por una serie de garantías. Esta serie de garantías podrían resumirse de la siguiente manera: derecho al acceso a los órganos jurisdiccionales, derecho a un proceso con las garantías mínimas, derecho a una resolución fundada en derecho que ponga fin a la controversia y derecho a la efectividad de las resoluciones

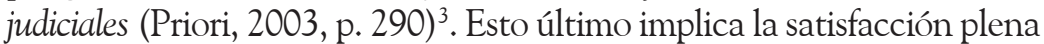
de la situación jurídica material lesionada o amenazada (Priori, 2003, p. 281 $)^{4}$. De esta manera, la efectividad de la tutela jurisdiccional se

2 En palabras de Giovanni Priori, «conforme al contenido de la tutela jurisdiccional efectiva, se hace necesario que la sentencia que se dicte al término del proceso pueda ser ejecutada, pues solo así se brinda una real y efectiva tutela a quienes inicien el proceso» (2009, pp. 284, 288).

3 Sobre este particular, el profesor Priori ha mencionado que «resulta pues manifiesta la expresión de nuestro máximo intérprete de la Constitución de que los derechos necesitan ser efectivos y que para ello se requiere de la protección jurisdiccional de los mismos» (Priori, 2003, p. 284).

$4 \mathrm{Al}$ respecto, nos parece relevante la siguiente sentencia expedida por el Tribunal Constitucional: «en ese sentido, el Tribunal Constitucional debe recordar que el derecho constitucional a la tutela jurisdiccional, reconocido en el inciso 3 del artículo 139 de la Constitución, no solamente consiste 
encuentra estrechamente vinculada con la instrumentalidad misma del proceso (Priori, 2003, p. 281).

La efectividad de la tutela jurisdiccional constituye el rasgo esencial de la tutela jurisdiccional efectiva. Así, una tutela que no fuera efectiva, por definición, no sería tutela. Bajo dicha premisa, es posible excusar la grave omisión del constituyente consistente en no incorporar el rasgo de la «efectividad» del derecho a la tutela jurisdiccional en el texto constitucional, puesto que una tutela jurisdiccional que no es efectiva no es en realidad una auténtica tutela (Priori, 2003, p. 281).

Al respecto, el Tribunal Constitucional peruano expresó lo siguiente:

A diferencia de los que sucede en otras constituciones, la nuestra no alude al derecho a la tutela jurisdiccional «efectiva». Sin embargo, en modo alguno puede concebirse que nuestra Carta Fundamental tan solo garantice un proceso «intrínsecamente correcto y leal, justo sobre el plano de las modalidades de su tránsito, sino también (...) capaz de consentir los resultados alcanzados, con rapidez y efectividad» (Colegio de Abogados de Ica c. decreto de urgencia, fundamento 9).

En consecuencia, podemos concluir que la efectividad es el rasgo fundamental de la tutela jurisdiccional y que dicho atributo permite que el proceso alcance la instrumentalidad que está llamado a cumplir en un Estado Constitucional de Derecho.

En ese sentido, una sentencia será efectiva cuando, luego de que la misma ha sido obtenida como consecuencia de un debido proceso, el mandato judicial que ella contiene se vea materializado en la realidad ${ }^{5}$. En otras palabras, la pretensión nunca queda satisfecha con la decisión que declare que la pretensión es fundada, sino cuando lo ordenado en la sentencia o el laudo sea verdaderamente cumplido ${ }^{6}$. El principio de efectividad exige una auténtica concreción, no solo a través del

en el derecho de acceder a un tribunal de justicia en forma libre, sin que medien obstáculos que impidan o disuadan irrazonablemente su acceso, que sea independiente y se encuentre previamente determinado por la ley, sino también que las resoluciones que los tribunales puedan expedir resolviendo la controversia o incertidumbre jurídica sometida a su conocimiento sean cumplidas y ejecutadas en todos y cada uno de sus extremos, sin que so pretexto de cumplirlas, se propicie en realidad una burla a la majestad de la administración de la justicia en general y, en forma particular, a la que corresponde a la justicia constitucional» (Jaime Mamerto Ramírez Benavides c. Ministerio del Interior, fundamento 4 f).

5 Al respecto, el profesor J esús Gonzáles ha expresado que «la tutela jurisdiccional no será efectiva si el mandato contenido en la sentencia no se cumple. La pretensión no quedará satisfecha con la sentencia que declare si está o no fundado, sino cuando lo mandado en la sentencia sea cumplido. Si la sentencia declara que la pretensión es conforme al ordenamiento jurídico y accede a lo pedido, la tutela jurisdiccional no será efectiva hasta que se efectúe el mandato judicial y el que accionó obtenga lo pedido» (2001, p. 337). En la misma línea, el Tribunal Constitucional peruano se ha expresado que «el derecho a la efectividad de las resoluciones judiciales garantiza que lo decidido en una sentencia se cumpla, y que la parte que obtuvo un pronunciamiento de tutela, a través de la sentencia favorable, sea repuesta en su derecho y compensada, si hubiere lugar a ello, por el daño sufrido» (Colegio de Abogados de Ica c. decreto de urgencia, fundamento 11).

6 Al respecto, el profesor de O livera ha señalado textualmente que «en el plano del derecho procesal, la cuestión tiene que ver con la efectividad del instrumento procesal, tanto en términos de tiempo como

NULLA EXECUTIO SINETITULO: EL ESCRUTINIO DE LOS LAUDOS EN LA PRÁCTICA DEL ARBITRAJE INTERNACIONAL COMO

MECANISMO

PARA OPTIMIZAR LA EJECUCIÓN DE LOS LAUDOS EN EL PERÚ

NULLA EXECUTIO SINE TITULO: THE SCRUTINY OF AWARDS IN THE PRACTICE OF INTERNATIONAL ARBITRATION AS A MECHANISM TO OPTIMIZE THE ENFORCEMENT OF AWARDS IN PERU 
pronunciamiento judicial que declara o constituye el derecho o impone la condena, sino mediante su efectivización o realización material, la cual se logra mediante el cumplimiento de la sentencia o el laudo en sus propios términos (Livy Margot Chumacero Marticorena c. Gobierno Regional de Piura, fundamento 11).

Bajo este marco, se han planteado cuatro grados de efectividad. Los dos grados que nos interesan por el momento son la efectividad de segundo grado y la de cuarto grado: es decir, que la decisión jurisdiccional resuelva sobre todas las pretensiones sometidas a la jurisdicción (efectividad de segundo grado) y que dicha decisión sea ejecutada en sus términos exactos y completos de manera coercitiva ante la falta de cumplimiento espontáneo (efectividad de cuarto grado) ${ }^{7}$.

El principio de efectividad de cuarto grado determina entonces que los laudos y las sentencias deben ser susceptibles de ejecución coercitiva. Para nuestro análisis, serán relevantes los laudos de condena, como, por ejemplo, los laudos de condena relativos a una deuda dineraria. En efecto, los laudos pueden ser declarativos, constitutivos o de condena. Los laudos declarativos solo reconocen una determinada situación o relación jurídica; los constitutivos, por el contrario, modifican, constituyen o extinguen una relación o situación jurídica; en cambio, los de condena disponen la realización de una determinada conducta por la parte demandada (Priori, 2009, p. 283).

En suma, la efectividad en cuarto grado del proceso arbitral se mide en función de la ejecución de los laudos de condena, pues solo en estos casos nos referimos a conductas puntuales exigibles al obligado y que son susceptibles de ejecución coercitiva directa.

\section{III.EL DERECHO A LA EJECUCIÓN DE LAS DECISIONES JURISDICCIONALES}

En el ordenamiento jurídico peruano, el derecho a la ejecución de las decisiones jurisdiccionales se encuentra previsto en el inciso 2 del artículo 139 de la Constitución, el cual señala expresamente lo siguiente:

de contenido de los pronunciamientos judiciales, con miras a lograr la justicia del caso concreto, y con observancia de las garantías del debido proceso legal» (2007, p. 156).

7 Para Chamorro, le efectividad se mide en cuatro grados: «La efectividad de primer grado garantiza a los ciudadanos la obtención de una respuesta del órgano jurisdiccional. Queda claro entonces que la tutela jurisdiccional efectiva no se agota en el mero acceso y en el proceso debido, sino que se requiere además una respuesta del órgano jurisdiccional. La efectividad del segundo grado garantiza que la resolución del órgano jurisdiccional será una que resuelva el problema planteado. Sin embargo, esto no quiere decir que este derecho garantice a los ciudadanos un tipo especial de respuesta jurisdiccional, sino solo que se resuelva el problema planteado independientemente de la respuesta que se dé, siempre que, claro está, dicha solución sea razonable y esté en armonía con el ordenamiento jurídico. La efectividad de tercer grado garantiza que la solución al problema planteado sea razonable y extraída del ordenamiento jurídico; la efectividad de cuarto grado garantiza que la decisión adoptada por un órgano jurisdiccional será ejecutada» (Chamorro, 1994, pp. 277-279). 
Artículo 139.- Son principios y derechos de la función jurisdiccional: [...]

2.- La independencia en el ejercicio de la función jurisdiccional. Ninguna autoridad puede avocarse a causas pendientes ante el órgano jurisdiccional ni interferir en el ejercicio de sus funciones. Tampoco puede dejar sin efecto resoluciones que han pasado en autoridad de cosa juzgada, ni cortar procedimientos en trámite, ni modificar sentencias ni retardar su ejecución (las cursivas son nuestras).

Por su parte, el artículo 4 de la Ley Orgánica del Poder Judicial se expresa en el mismo sentido:

No se puede dejar sin efecto resoluciones judiciales con autoridad de cosa juzgada, ni modificar su contenido, ni retardar su ejecución, ni cortar procedimientos en trámite, bajo responsabilidad política, administrativa, civil, penal que la ley determine en cada caso (las cursivas son nuestras).

Como se puede observar, conforme a los preceptos enunciados, es imprescindible que el laudo o sentencia que se dicte al término de un proceso sea ejecutado de la forma más célere posible, ya que es clara la alusión de la Constitución al «retardo» como un estado de cosas inconstitucional (Priori, 2009, p. 284) . $^{8}$.

$\mathrm{Al}$ respecto, resulta preciso recordar que el Tribunal Constitucional peruano ha expresado que el derecho a la ejecución de las decisiones es una manifestación del derecho a la tutela jurisdiccional efectiva. Sobre el particular, el máximo intérprete de la Constitución señaló expresamente lo siguiente:

El derecho a la ejecución de las resoluciones judiciales que han pasado en autoridad de cosa juzgada, es una manifestación del derecho a la tutela jurisdiccional, reconocido en el inciso 3) del artículo 139 de la Constitución. También se encuentra aludido en el segundo párrafo del inciso 2) del artículo 139, cuando se menciona que "ninguna autoridad puede (...) dejar sin efecto resoluciones judiciales que han pasado en autoridad de cosa juzgada (...) ni retardar su ejecución (Colegio de Abogados de Ica c. decreto de urgencia, fundamento 8).

8 De igual manera, el Tribunal Constitucional español ha señalado reiteradamente lo siguiente: «el derecho a la tutela judicial efectiva reconocido en el artículo 24.1, CE, comprende, entre otros, el derecho a que sean ejecutadas en sus propios términos las resoluciones judiciales firmes, pues sin ello la tutela de los derechos e intereses legítimos de los que obtuvieron una resolución favorable no sería efectiva, sino que se quedaría en unas declaraciones de intenciones y de reconocimiento de derechos sin alcance práctico, correspondiendo a los Tribunales velar por ese cumplimiento, como expresamente se declara por el artículo 117.3, CE, de modo que desconoce el derecho fundamental el J uez que, por omisión, pasividad o defectuoso entendimiento, se aparta, sin causa justificada, de lo previsto en el fallo que debe ejecutarse, o se abstiene de adoptar las medidas necesarias para su ejecución, cuando le sea legalmente exigible» (Angeles García Barroso c. Juzgado de lo Social de Sevilla, fundamento $2 \mathrm{~A}$ ).

\section{NULLA EXECUTIO SINE TITULO: EL ESCRUTINIO DE LOS LAUDOS EN LA PRÁCTICA DEL ARBITRAJE INTERNACIONAL COMO \\ MECANISMO \\ PARA OPTIMIZAR LA EJECUCIÓN DE LOS LAUdOS EN EL PERÚ}

NULLA EXECUTIO SINE TITULO:THE SCRUTINY OF AWARDS IN THE PRACTICE OF

INTERNATIONAL ARBITRATION AS A MECHANISM TO OPTIMIZE THE ENFORCEMENT OF AWARDS IN PERU 
De igual manera, la Corte Europea de los Derechos Humanos expresó, en el emblemático caso Hornsby c. Grecia, lo siguiente: «El derecho de acceso a los tribunales sería ilusorio si el ordenamiento jurídico interno de un Estado miembro permitiera que una decisión definitiva y obligatoria quedase inoperativa en detrimento de la parte. Por tal razón, la ejecución de la decisión, sea cual fuere la jurisdicción, debe ser considerada como parte integrante del proceso» (citado en de Oliveira, 2007, p. 160).

La ejecución es, entonces, una expresión del principio de efectividad de cuarto grado a través del cual el propio Estado garantiza a los ciudadanos el recurso a su ius imperium con el propósito de ejecutar coercitivamente las sentencias. Y, en el caso del arbitraje, garantiza que los laudos sean ejecutados mediante el auxilio de la jurisdiccional judicial, en vista de que la jurisdiccional arbitral carece de coertio (véanse artículo 8, inciso 3 y artículo 67 de la Ley de Arbitraje, en los que se regula el auxilio judicial para la ejecución de laudos artículos).

\section{IV.LA APLICACIÓN INMEDIATA DEL ARTÍCULO 139 DE LA CONSTITUCIÓN AL ARBITRAJE}

Si bien en las secciones anteriores hemos asumido que las garantías procesales previstas en el artículo 139 de la Constitución son de plena aplicación al arbitraje, debemos advertir que esta es una cuestión sumamente controvertida en la doctrina. Esta discusión, ciertamente, no es fútil, puesto que dependiendo de la posición que se adopte se concluirá si garantías procesales como la defensa, la motivación y la efectividad de las decisiones son imperativas también en el arbitraje.

En nuestra opinión, las garantías procesales enmarcadas en el artículo 139 de la Constitución son de aplicación inmediata al arbitraje, siempre que no contravengan su naturaleza (véase Rivarola, 2006). Sería un despropósito pensar, por ejemplo, que el derecho a la doble instancia, el derecho a un juez predeterminado por la ley o la publicidad de los procesos son de aplicación inmediata al arbitraje cuando prácticamente todas las leyes o reglamentos de arbitraje del mundo reconocen que los laudos son inapelables, que el derecho a designar un árbitro es un derecho fundamental y que la confidencialidad es inherente al arbitraje? Resulta también controvertido el caso del derecho a la motivación de las decisiones jurisdiccionales, puesto que nuestra la Ley de Arbitraje permite a las partes pactar en contra de la motivación y es difícil pensar

9 Este último punto es quizá el más controvertido. Hay quienes sostienen que la confidencialidad es inherente al arbitraje. De lo que no cabe la menor duda es que los procesos arbitrales no son públicos. 
en un derecho fundamental dispositivo. Los derechos fundamentales son - por definición - irrenunciables ex ante.

Ahora bien, existen sin duda otras garantías como la imparcialidad, la defensa, la cosa juzgada o la efectividad que son de plena aplicación al arbitraje. No es posible imaginar un proceso arbitral sin la vigencia de estas garantías. Ciertamente, el arbitraje permite que las partes diseñen un procedimiento que se adecue a sus necesidades y expectativas e incluso les permite evitar los formalismos propios de un proceso judicial. Sin embargo, ese procedimiento particular debe respetar garantías mínimas reconocidas en la Constitución (Palacios, 2009, p. 251).

Sobre este particular, el profesor Reynaldo Bustamante se ha pronunciado en el mismo sentido, con las siguientes palabras:

¿Rigen entonces los derechos fundamentales en el arbitraje? Por supuesto que sí. Sin embargo, no han faltado voces que rechazan esta afirmación bajo el argumento de que la virtualidad de los derechos fundamentales es una cuestión que debe asegurarse en el marco de las relaciones con el Estado, con el fin de que protejan a los particulares de los excesos que pueda cometer un poder exorbitante como el estatal, pero que no tendría sentido extenderla — por lo menos no de la misma manera - en el marco de las relaciones entre privados, como es el caso del arbitraje, porque en estas esa disparidad desaparece, o por lo menos se ve atenuada por la autonomía de la voluntad (Bustamante, 2013, p. 397).

En el mismo sentido, como señala Palacios, el Tribunal Constitucional estableció (en la sentencia del caso Fernando Cantuarias Salaverry c. Corte Superior de Justicia de Lima) lo siguiente:

El enmarcamiento del arbitraje dentro de la Constitución, lejos de generar un perjuicio para la institución arbitral, es sumamente beneficioso, pues pone fin a las especulaciones e incertidumbres que se presentaban en curso de los arbitrajes y, a consecuencia de ello, pone coto al posible abuso de recurrencia judicial para que, por iniciativa de las partes, los jueces, fiscales y autoridades administrativas pretendan suspender, interrumpir los procesos arbitrales en curso, lo que sería peor, revisar a su arbitrio el contenido de los laudos (Palacios, 2009, p. 252).

Finalmente, la aplicación directa de los derechos fundamentales al arbitraje ha sido corroborada por el Tribunal Constitucional en la sentencia del caso Fernando Cantuarias Salaverry c. Corte Superior de Justicia de Lima, en la que señaló expresamente que «si bien la autonomía de la jurisdicción arbitral tiene consagración constitucional, no lo es menos que, como cualquier particular, se encuentra obligada a respetar los derechos fundamentales» (fundamento 20).

NULLA EXECUTIO

SINE TITULO: EL

ESCRUTINIO DE

LOS LAUDOS EN

LA PRÁCTICA

DEL ARBITRAJE

INTERNACIONAL

COMO

MECANISMO

PARA OPTIMIZAR

LA EJECUCIÓN DE

LOS LAUDOS EN

EL PERÚ

NULLA EXECUTIO

SINE TITULO:THE

SCRUTINY OF

AWARDS IN THE

PRACTICE OF

INTERNATIONAL

ARBITRATION AS

A MECHANISM

TO OPTIMIZE THE

ENFORCEMENT

OF AWARDS IN

PERU 
Por ninguna razón debemos pensar que, al exigir que el arbitraje respete derechos fundamentales - como el derecho a la defensa, el derecho a la prueba o el derecho a la efectividad de las decisiones jurisdiccionales-, se deberían multiplicar las exigencias de los formalismos procesales, de manera tal que se convierta al proceso arbitral en el hermano mellizo del proceso judicial (Palacios, 2009, p. 251). Nada de eso. Simplemente consideramos que no existe ninguna disciplina que se encuentre al margen de la Constitución y, más allá de que el arbitraje encuentre sustento en la autonomía privada, el arbitraje no es la excepción.

En consecuencia, el reconocimiento del arbitraje como mecanismo de resolución de conflictos comporta la aplicación a los tribunales arbitrales de las normas constitucionales que no contravengan su naturaleza. En la práctica del arbitraje internacional, no es muy usual que los árbitros invoquen garantías contenidas en un texto constitucional, no solo porque muchas de ellas no son aplicables al arbitraje, sino porque los árbitros internacionalmente reconocidos suelen saber muy bien qué actos se encuentran dentro de los límites del debido proceso y cuáles no. Normalmente no necesitan estudiar los desarrollos jurisprudenciales de cada una de las garantías procesales para advertir que cierto acto vulnera el derecho de defensa, por ejemplo.

Sin perjuicio de lo anterior, siendo el derecho a la ejecución de las decisiones jurisdiccionales de plena aplicación al arbitraje, no cabe la menor duda de que cualquier particular tiene derecho a exigir al Estado que provea todos los medios adecuados para que se garantice plenamente la ejecución del laudo.

\section{LA EJECUCIÓN DEL LAUDO Y SUS TROPIEZOS \\ La efectividad del laudo puede derivar del propio comportamiento de la parte perdedora en el proceso, quien voluntariamente puede aceptar cumplirlo, situación que se presenta como deseable ${ }^{10}$. Sin embargo, si el sujeto demandado no cumple voluntariamente con lo ordenado en el laudo, se deben poner en práctica los diversos mecanismos previstos por el ordenamiento jurídico para ejecutar lo establecido en el laudo, procediéndose en estos casos a su ejecución (Priori, 2009, p. 285) ${ }^{11}$.}

10 En relación con este punto, se ha señalado lo siguiente: «dicha situación se deriva del deber general del cumplimiento de las sentencias que deriva del reconocimiento del derecho a la tutela jurisdiccional efectiva. De esta manera, todos deben prestar esta colaboración, y los afectados concretamente por el fallo vienen ineludiblemente obligados a su cumplimiento, cualquiera sea la persona a la que se refiere el mandato judicial» (Ortega, 2001, pp. 449ss.).

11 Sobre este particular, el profesor González ha expresado que «la prestación de justicia no sería efectiva si el mandato de la sentencia no fuera cumplido. El derecho a la tutela jurisdiccional efectiva - que suelen reconocer todas las Constituciones- comprende no solo la facultad para exigir y obtener una sentencia que decida si la pretensión está o no fundada, sino que lo que en ella se ha resuelto sea llevado a efecto, con, sin o contra la voluntad del obligado. Los Tribunales han de juzgar y hacer ejecutar lo juzgado» (2001, p. 425). 
En ese sentido, si el obligado se resiste de cualquier manera a cumplir lo ordenado en el laudo, el Estado - que prohíbe la autodefensadebe emplear los medios necesarios para superar dicha resistencia, legitimando la utilización de la fuerza para lograrlo (González, 2001, p. 337). El derecho a la ejecución de las decisiones jurisdiccionales exige entonces que el juez disponga de los instrumentos para garantizar que el demandante obtenga el bien de la vida (la utilidad) que el laudo ha reconocido.

Al respecto, el profesor González se ha pronunciado del siguiente modo: «Si el obligado no cumple lo mandado por la sentencia, el derecho a la tutela judicial efectiva comprende la facultad de dirigirse a un órgano jurisdiccional para que adopte cuantas medidas y providencias fuesen necesarias para que se realice lo dispuesto en el fallo» (2001, p. 341).

Sin embargo, con frecuencia, estas medidas y providencias pueden ser insuficientes y, a pesar de los esfuerzos, el derecho de ejecución tropieza con varios obstáculos. En muchos casos, esto sucede ante la inejecución pura y simple del laudo o incluso también ante la alteración de los términos del mismo. Estos supuestos hipotéticos son muy peligrosos. Sin duda, la inejecución pura y simple supondría ignorar los derechos e intereses de la parte que obtuvo tutela efectiva a través de una decisión favorable (Coto Minero c. Audiencia Territorial de Valladolid). A su vez, la hipotética posibilidad de alterar los términos de los laudos a ejecutar ocasionaría una gravísima inseguridad jurídica (Obando, 2011, p. 1999). Imaginemos, por ejemplo, un supuesto en el cual en sede de ejecución el juez determina los intereses sin utilizar ningún parámetro brindado por el tribunal arbitral. Ello no solo vulneraría la autonomía privada de las partes, las cuales nunca consintieron que el juez en ejecución realice semejante tarea, sino que incluso su derecho a la defensa se vería menoscabado, pues no podrían contradecir ni probar con la misma amplitud que en un proceso cognitivo.

Lo cierto es que la efectividad de los laudos en el ordenamiento jurídico peruano se ha visto relativizada y ha tenido tropiezos adicionales. Sucede que, en múltiples casos, los árbitros no determinan con claridad los conceptos que integran las obligaciones contenidas en los laudos, lo que ocasiona que en reiteradas ocasiones la parte vencida cuestione la ejecutabilidad del laudo y, en consecuencia, la efectividad del mismo se vea relativizada (Julio Huamán Gómez c. Corporación José R. Lindley). En otras palabras, lo que sucede con frecuencia es que los conceptos contenidos en los laudos no se encuentran correctamente determinados y, por ende, se imposibilita su ejecución.

La naturaleza del proceso de ejecución en general, y de la ejecución de laudos arbitrales en particular, exige que la suma dineraria contenida en este sea líquida o liquidable mediante operación aritmética. En efecto,

NULLA EXECUTIO

SINE TITULO: EL

ESCRUTINIO DE

LOS LAUDOS EN

LA PRÁCTICA

DEL ARBITRAJE

INTERNACIONAL

COMO

MECANISMO

PARA OPTIMIZAR

LA EJECUCIÓN DE

LOS LAUDOS EN

EL PERÚ

NULLA EXECUTIO

SINE TITULO: THE

SCRUTINY OF

AWARDS IN THE

PRACTICE OF

INTERNATIONAL

ARBITRATION AS

A MECHANISM

TO OPTIMIZE THE

ENFORCEMENT

OF AWARDS IN

PERU 
el artículo 689 del Código Procesal Civil establece expresamente lo siguiente:

\section{Artículo 689.- Requisitos Comunes}

Procede la ejecución cuando la obligación contenida en el título es cierta, expresa y exigible. Cuando la obligación es de dar suma de dinero, debe ser, además, líquida o liquidable mediante operación aritmética.

En el mismo sentido, es preciso recordar que si la suma es liquidable mediante simple operación aritmética, el ejecutante debe acompañar una liquidación realizada de acuerdo con los criterios establecidos en el laudo por el tribunal arbitral. Al respecto, el artículo 717 del Código Procesal Civil fija la siguiente regla:

Artículo 717.- Ejecución de suma ilíquida

Si el título de ejecución condena al pago de cantidad ilíquida, el vencedor debe acompañar liquidación realizada siguiendo los criterios establecidos en el título o en su defecto los que la ley disponga.

La liquidación contenida en el mandato de ejecución puede ser observada dentro del tercer día, luego de lo cual el Juez resolverá aprobándola o no, en decisión debidamente fundamentada.

Lo anterior significa que el tribunal arbitral debe haber establecido previamente ya sea el quantum específico o al menos el lineamiento claro e inobjetable que llegue a la suma a ejecutar mediante una simple operación aritmética. Prescindir de dicho requisito implicaría que la jurisdicción estatal tome decisiones respecto a las pretensiones del proceso arbitral, lo que implicaría vulnerar una competencia ajena.

Sobre este particular, es preciso recordar que el artículo 68 de la Ley de Arbitraje establece condiciones procesales específicas para ejecutar un laudo arbitral:

Artículo 68.- Ejecución judicial

1. La parte interesada podrá solicitar la ejecución del lando ante la autoridad judicial competente acompañando copia de este y de sus rectificaciones, interpretaciones, integraciones y exclusiones y, en su caso, de las actuaciones de ejecución efectuada por el tribunal arbitral.

2. La autoridad judicial, por el solo mérito de los documentos referidos en el numeral anterior, dictará mandato de ejecución para que la parte ejecutada cumpla con su obligación dentro de un plazo de cinco (5) días, bajo apercibimiento de ejecución forzada (las cursivas son nuestras).

Como se puede apreciar, en los casos en que los conceptos contenidos en los laudos no se encuentran correctamente determinados, no es posible abrir, en medio del proceso de ejecución, discusión, probanza ni 
determinación alguna respecto de los montos a ser ejecutados. Como es bien sabido, en los proceso de ejecución la autoridad judicial solo debe intervenir para ejecutar lo que se encuentra en el título ejecutivo de modo cierto, claro, expreso, exigible y líquido (o liquidable) (Ley de Arbitraje, artículo 68). El laudo debe poder ser ejecutable por su solo mérito, sin necesidad de que se realice ningún examen adicional.

Así, por ejemplo, en el caso de los intereses, el tribunal arbitral debe indicar la tasa y el momento desde el cual empiezan a generarse, convirtiendo a la suma en liquidable. El juez no debe tomar ninguna decisión, pues su labor se reduce a ejecutar lo ya decidido por el tribunal arbitral. Similar criterio es aplicable a los costos del proceso arbitral ${ }^{12}$, los cuales deben ser fijados por el tribunal arbitral; o, al menos, el tribunal arbitral debe proporcionar parámetros objetivos, claros y concretos a fin de que, en el proceso de ejecución, el juez, sin realizar ningún tipo de examen, pueda calcular los costos en base a una operación aritmética simple.

En definitiva, para que las obligaciones de dar suma de dinero puedan constituir título ejecutivo, el título debe ser líquido o liquidable. Asimismo, para su respectiva ejecución, han de seguirse los criterios establecidos en el título o los que la ley disponga. Los jueces no pueden suplir una competencia que corresponde exclusivamente a los árbitros en virtud de un convenio arbitral.

\section{VI.LOS PROBLEMAS DE LAEJECUCIÓN DEL LAUDO EN LA JURISPRUDENCIA}

Un ejemplo en el que la ejecución del laudo se encontró con una serie de tropiezos fue el caso Julio Huamán Gómez c. Corporación José R. Lindley, seguido ante la Segunda Sala Civil con Subespecialidad Comercial. En dicho caso, la Corporación José R. Lindley sostenía que la ejecución que pretendía Julio Huamán Gómez SCRL debía ser desestimada, puesto que la obligación contenida en el laudo relativa a las costas arbitrales no estaba determinada.

12 Es oportuno precisar que las costas se encuentran constituidas por las tasas judiciales, los honorarios de los órganos del auxilio judicial y los demás gastos judiciales realizados en el proceso. Se trata, pues, de un concepto inherente al proceso judicial y no al proceso arbitral. El artículo 70 de la Ley de Arbitraje establece lo siguiente: «el tribunal arbitral fijará en el laudo los costos del arbitraje. Los costos del arbitraje comprenden: a) los honorarios y gastos del tribunal arbitral; b) los honorarios y gastos del secretario; c) los gastos administrativos de la institución arbitral; d) los honorarios y gastos de los peritos o de cualquier otra asistencia requerida por el tribunal arbitral; e) los gastos razonables incurridos por las partes para su defensa en el arbitraje; f) los demás gastos razonables originados en las actuaciones arbitrales». Asimismo, el artículo 69 de la Ley de Arbitraje establece lo siguiente: «las partes tienen la facultad de adoptar, ya sea directamente o por referencia a reglamentos arbitrales, reglas relativas a los costos del arbitraje. A falta de acuerdo, el tribunal arbitral dispondrá lo conveniente, con sujeción a lo dispuesto en este título».

NULLA EXECUTIO SINE TITULO: EL ESCRUTINIO DE LOS LAUDOS EN LA PRÁCTICA DEL ARBITRAJE INTERNACIONAL COMO

MECANISMO

PARA OPTIMIZAR LA EJECUCIÓN DE LOS LAUDOS EN EL PERÚ

NULLA EXECUTIO SINE TITULO: THE SCRUTINY OF AWARDS IN THE PRACTICE OF INTERNATIONAL ARBITRATION AS A MECHANISM TO OPTIMIZE THE ENFORCEMENT OF AWARDS IN PERU 
En su fallo, los vocales de la Segunda Sala Civil con Subespecialidad Comercial consideraron que todos los conceptos a ejecutar habían sido correctamente determinados, salvo el último, correspondiente a los costos arbitrales sobre cuya determinación no se había señalado lineamiento alguno. En tal sentido, los vocales concluyeron que los costos arbitrales no podían ser objeto de ejecución por lo siguiente:

6.1. Como se puede advertir, los conceptos señalados son todos determinados (o contienen las pautas mínimas para su determinación, como los intereses), salvo el último, correspondiente a los costos arbitrales, sobre cuya determinación no se ha señalado lineamiento alguno.

6.2. Por ello, conforme a lo señalado anteriormente, este concepto (costos arbitrales) no puede ser objeto de ejecución en el presente proceso de ejecución de laudo arbitral, pues no reúne la citada condición esencial para ser encaminado a través de esta vía (Julio Huamán Gómez c. Corporación José R. Lindley).

Como última precisión, los vocales destacaron que la indeterminabilidad de los costos arbitrales no debía repercutir en ninguno de los otros conceptos que integraban la obligación, de modo tal que resultaba viable el reclamo del pago de dichos conceptos en la vía ejecutiva, pues estos habían sido claramente determinados (Julio Huamán Gómez c. Corporación José R. Lindley, 7).

Otro caso emblemático es Suring SAC c. Ministerio de Educación, seguido ante la Segunda Sala Civil con Subespecialidad Comercial, en el que la ejecución del laudo sufrió nuevos tropiezos. En dicho caso, el Ministerio de Educación cuestionó la ejecutabilidad del laudo, sosteniendo que los conceptos contenidos en él no eran líquidos ni determinados. En otras palabras, el demandado señaló que las estimaciones contenidas en el laudo no eran lo suficientemente precisas o claras como para que el mismo pueda constituir título ejecutivo.

En su fallo, los vocales advirtieron que, si bien los árbitros habían declarado fundadas varias pretensiones, ordenando que la ejecutada pague al ejecutante montos por distintos conceptos (gastos generales, penalidades, diferencia de valorizaciones, entre otros), no todos los conceptos habían sido precisados en cuanto al monto que representaban.

En tal sentido, los vocales verificaron si en el propio título ejecutivo se podía identificar los indicadores referenciales o parámetros a utilizar para liquidar la cuantía de los conceptos a ejecutar. Sin embargo, los vocales advirtieron que, si bien había algunos conceptos determinados por el laudo, no se había dicho nada respecto de la tasa de interés aplicable, ni tampoco del período dentro del cual debían ser estimados, ni si estos eran capitalizados o capitalizables. 
Sobre este particular, la Segunda Sala Civil con Subespecialidad Comercial señaló en su fallo expresamente lo siguiente:

Las estimaciones contenidas en el laudo no son del todo precisas o claras, pues no se sabe si corresponden a montos ya establecidos por el propio Tribunal, o si sobre ellos debe realizarse nuevas estimaciones, como en la sexta pretensión subordinada, relacionada con los intereses de las valorizaciones, se ha establecido un monto, pero no se dice nada sobre el período al que corresponde dicho monto, haciendo inviable un cálculo correcto de los intereses que se generarían hasta la fecha de pago.

En otros extremos, es el contenido de la pretensión que ha sido declarada fundada la que no se identifica con claridad, como en la sétima pretensión subordinada, que versa sobre los intereses generados por la devolución del fondo de garantía. En este caso habría que establecer si ello incluye solo los intereses o también la devolución de dicho fondo, o si este fondo ya fue devuelto y solo restan los intereses" (Suring SAC c. Ministerio de Educación, 3).

Así, pese a las deficiencias advertidas en la determinación de cada uno de los conceptos reconocidos en el laudo, la ejecutante solicitó a través de su demanda el pago del monto de S/. 408,691.11, señalando que dicha cantidad sería el resultado de la suma de todos los conceptos que han sido reconocidos por el laudo. Tal resultado ha sido calculado agregando a cada pretensión el IGV y los intereses, pese a que tales conceptos no han sido reconocidos por todas las pretensiones resueltas en el laudo. De igual modo, incluye en su cálculo conceptos cuyo reconocimiento no es del todo claro (ej. devolución de fondo) (Suring SAC c. Ministerio de Educación, 5).

NULLA EXECUTIO SINETITULO: EL ESCRUTINIO DE LOS LAUDOS EN LA PRÁCTICA DEL ARBITRAJE INTERNACIONAL COMO

MECANISMO

PARA OPTIMIZAR LA EJECUCIÓN DE LOS LAUDOS EN EL PERÚ

NULLA EXECUTIO SINE TITULO: THE SCRUTINY OF AWARDS IN THE PRACTICE OF INTERNATIONAL ARBITRATION AS A MECHANISM TO OPTIMIZE THE ENFORCEMENT OF AWARDS IN PERU

En virtud de dichas consideraciones, los vocales concluyeron que las deficiencias identificadas debían ser corregidas a través del dictado de un nuevo pronunciamiento arbitral, verificando previamente de manera seria y clara los alcances del título ejecutivo puesto a cobro, el mismo que debe cumplir con todas y cada una de las condiciones que la ley exige (obligación cierta, expresa, exigible, líquida o liquidable).

Por otra parte, resulta especialmente pertinente el caso Gerencia Comercial Inmobiliaria c. Electrocentro, en el que una vez más la efectividad del laudo se tropezó con nuevos obstáculos. En dicho caso, la parte ejecutada cuestionó la ejecutabilidad del laudo, sosteniendo que el laudo no contenía una suma líquida. Al respecto, la Segunda Sala Civil con Subespecialidad Comercial se pronunció de la siguiente manera:

Es así que ni siquiera es factible analizar dentro de la totalidad del fallo arbitral, la existencia de conceptos susceptibles de ser liquidados o no mediante operación aritmética simple (muchos de ellos no lo son, 
de una simple revisión, como se ha señalado; por ejemplo, de los párrafos transcritos relativos a los puntos controvertidos 23, 24, 36 y 37, se observa que no hay pautas de esa naturaleza para poder alcanzar la cifra inobjetable a ejecutar con solo operación aritmética), por cuanto no se ha distinguido qué conceptos o extremos del laudo arbitral son los cedidos" (Gerencia Comercial Inmobiliaria c. Electrocentro, 6).

Como se puede apreciar, los vocales concluyeron que el título que se pretendía ejecutar no cumplía con los requisitos legales que permiten la ejecución del laudo, puesto que únicamente se pueden ejecutar obligaciones expresas, líquidas (o liquidables, según lo expuesto), ciertas y exigibles, por lo que se declaró improcedente la demanda.

De igual manera, en el caso Gerardo Widauski c. Electrocentro, la Segunda Sala Civil con Subespecialidad Comercial expresó que

3.2. [...] la determinación del tipo de interés y de la tasa no corresponde a la etapa de ejecución, en la que solo se liquida lo que ya está establecido con precisión en la etapa previa (hasta el auto final).

3.3. Es por ello que al emitir la apelación el Sr. Juez A quo ha incurrido en vicio insalvable, debiendo declararse nula la resolución 03 en el extremo relativo a los intereses, a fin de que el Magistrado proceda a analizar el título de ejecución y de acuerdo a él volver a pronunciarse respecto del extremo señalado (Gerardo Widauski c. Electrocentro).

Sin perjuicio de lo anterior, resulta preciso señalar que existe al menos una sentencia que ha adoptado una posición opuesta a los casos anteriormente citados. A pesar de que es bien sabido que en un proceso de ejecución no puede someterse a debate o probanza las sumas a ser ejecutadas, ha existido al menos en un caso en que se ha sostenido una tesis contraria.

Así, en el caso Consorcio Recupera Callao c. Municipalidad Provincial del Callao, el ejecutado objetó la ejecutabilidad del laudo, señalando que «a) el monto ordenado pagar en el laudo arbitral asciende a S/.646,725.47 nuevos soles y no a lo ordenado por el juez que es de S/. 740,925.47 nuevos soles, pretendiéndose cobrar sumas distintas a las ordenadas pagar por el Tribunal Arbitral; b) el ejecutor del laudo no se encuentra facultado para presentar la liquidación de intereses en forma unilateral y arbitraria» (Consorcio Recupera Callao c. Municipalidad Provincial del Callao, 2).

Sin embargo, la Primera Sala Civil con Subespecialidad Comercial se pronunció en los siguientes términos:

El ejecutante no ha tomado en cuenta que conforme a la liquidación de fojas 70 - presentada por la parte ejecutante— se incorporaron a la 
ejecución del laudo los conceptos de gastos, costos y costas ordenados a pagar en el punto sexto del mismo laudo, los que fueron estimados por el ejecutante en S/. 94,200.00 nuevos soles, siendo que este extremo no fue cuestionado por la parte ejecutada, ya que su «contradicción» propuesta fue rechazada, además se puede apreciar de su contenido que la ejecutada no formuló ninguna objeción de la naturaleza que ahora pretende incorporar a debate, es decir, no propuso oposición al pago de estos conceptos.

De otro lado, en la misma resolución el Juez del proceso ha ordenado al ejecutado pagar a la ejecutante «los intereses devengados y por devengarse», por lo cual, no resulta cierto - como lo sostiene el apelante- que el juez haya ordenado el pago de una suma de dinero líquida por concepto de intereses, pues, si bien el ejecutante ha estimado este concepto en S/. 200,841.17 nuevos soles en la liquidación ya mencionada, el auto de pago no involucra el mismo, siendo este un concepto a establecerse finalmente en ejecución (Consorcio Recupera Callao c. Municipalidad Provincial del Callao, 5; las cursivas son nuestras).

Como no es difícil advertir, si bien existe una marcada tendencia mayoritaria que establece que no se pueden determinar los conceptos a ejecutar en ejecución, la cuestión no es absolutamente pacífica en la jurisprudencia. Existen posiciones distintas. En nuestra opinión, lo que está detrás de las jurisprudencias citadas es el resultado de una ponderación entre derechos fundamentales: autonomía de la voluntad, por un lado, y efectividad de las decisiones jurisdiccionales, por el otro.

Por un lado, se establece que no es admisible que, en un proceso de ejecución, sea posible que los jueces determinen los conceptos a ser ejecutados porque se alega que debe prevalecer la autonomía de voluntad de las partes, quienes (mediante la celebración de un convenio arbitral) han pactado que la decisión final de sus controversias debe estar en manos del tribunal arbitral y no de los jueces. A su vez, por otro lado se alega que los jueces sí están legitimados para determinar los conceptos en ejecución, con el propósito de salvaguardar el derecho a la efectividad de las decisiones jurisdiccionales.

\section{CONFLICTO DEPRINCIPIOS CONSTITUCIONALES}

En las jurisprudencias citadas, el problema con el que se han encontrado los jueces es que los tribunales arbitrales no habían cumplido debidamente con liquidar conceptos como las costas, los intereses, las penalidades, etcétera. En otras palabras, si bien en todos los casos las costas, los intereses y/o las penalidades habían sido articulados como pretensiones por los demandantes, las estimaciones de dichos conceptos efectuadas por el tribunal arbitral y contenidas en el laudo

NULLA EXECUTIO

SINE TITULO: EL

ESCRUTINIO DE

LOS LAUDOS EN

LA PRÁCTICA

DEL ARBITRAJE

INTERNACIONAL

COMO

MECANISMO

PARA OPTIMIZAR

LA EJECUCIÓN DE

LOS LAUDOS EN

EL PERÚ

NULLA EXECUTIO

SINE TITULO:THE

SCRUTINY OF

AWARDS IN THE

PRACTICE OF

INTERNATIONAL

ARBITRATION AS

A MECHANISM

TO OPTIMIZE THE

ENFORCEMENT

OF AWARDS IN

PERU 
no eran lo suficientemente precisas o claras como para que puedan constituir título ejecutivo.

En estas circunstancias, el juez se encuentra en una situación complicada: por un lado, emitir un pronunciamiento con respecto a dichos puntos implicaría violar una competencia ajena que le corresponde exclusivamente a los árbitros; mientras que, por otro lado, no pronunciarse con respecto a dichos puntos implica obligar a las partes a iniciar un nuevo arbitraje, asumir los costos del mismo y esperar años a que se dicte un nuevo laudo que se pronuncie sobre estos puntos. Este último escenario restringe, entre otros derechos, la efectividad de las decisiones jurisdiccionales.

En efecto, si es que el juez en ejecución realizase una cognición sumaria para determinar cualquiera de dichos conceptos, estaría restringiendo la autonomía privada de los contratantes y desnaturalizando el proceso de ejecución. Sin embargo, no ejecutar dichos conceptos implicaría restringir, entre otros derechos, la efectividad de las decisiones jurisdiccionales y la naturaleza del proceso en general, la cual implica resolver los conflictos de intereses, mas no dilatarlos.

Frente a este escenario, resulta pertinente resaltar que la potestad de los árbitros de resolver las controversias encuentra fundamento en la libertad y la autonomía de la voluntad de los particulares (Cremades, 2006, p. 187). El convenio arbitral, piedra angular del arbitraje, delimita la potestad decisoria de los árbitros. «Todo arbitraje se fundamenta sobre la voluntad de las partes en conflicto, existente o futuro, de tal manera que la cláusula arbitral es el punto neurálgico del arbitraje y su razón de ser» (Cremades, 2006, pp. 185-186) ${ }^{13}$. En este sentido, si un juez en ejecución de laudo se pronuncia sobre una pretensión que fue sometida a un proceso arbitral en virtud de un convenio arbitral, está desconociendo la autonomía de la voluntad de las partes.

En nuestra opinión, bajo ninguna circunstancia un juez puede suplir una competencia que les corresponde exclusivamente a los árbitros en virtud de un convenio arbitral. Sin embargo, no podemos negar que

13 Sobre este particular, el profesor Cremades ha señalado que «El arbitraje se justifica en la autonomía de la voluntad, fruto de la libertad, valor fundamental que nuestro ordenamiento jurídico propugna en el artículo 1.1 CE. Tal y como ha reconocido el Tribunal Constitucional, el arbitraje es “...un medio heterónomo de arreglo de controversias que se fundamenta en la autonomía de la voluntad de los sujetos privados (art. 1.1 CE)".

La piedra angular del arbitraje es el convenio arbitral; negocio jurídico por el que las partes expresan su voluntad de someter a arbitraje la solución de todas las cuestiones litigiosas, o de alguna de ellas, que se hayan planteado o que puedan plantearse respecto de una determinada relación jurídica [...]. La jurisdicción arbitral encuentra su fundamento en un negocio jurídico, el convenio arbitral, dirigido a la liquidación de una relación jurídica controvertida, de modo que la voluntad de las partes es la única fuente del arbitraje» (Cremades, 2006, pp. 187-188). 
el derecho a la ejecución impone a los órganos judiciales adoptar las medidas oportunas para garantizar la efectividad de las resoluciones ${ }^{14}$.

Pues bien, teniendo en cuenta que nos encontramos frente a un conflicto latente entre la autonomía privada y el derecho a la efectividad de las decisiones jurisdiccionales, la propuesta que compartiremos debe ser el resultado de una ponderación de principios constitucionales. En ese sentido, la solución debe propender a maximizar dichos principios constitucionales, puesto que, incluso en el ámbito de los derechos constitucionales, lo importante es maximizar.

En otro trabajo ya se ha señalado que

si bien los derechos fundamentales se encuentran en constante conflicto, ello no significa que un derecho debe ser sacrificado en desmedro de otro o que se debe elegir entre uno de los dos, sino que se debe optimizar a todos en su conjunto. Lo importante es comprender que lo que está detrás de la ponderación de derechos fundamentales es un análisis costo-beneficio. El juez tendrá que restringir algunos derechos y optimizar otros, pero siempre buscará la maximización de los derechos fundamentales en su conjunto (Núñez del Prado, 2016, p. 75).

Si bien uno de los remedios idóneos para resolver estos inconvenientes es el recurso de integración del laudo previsto en el artículo 57 (inciso 1, literal c) de la Ley de Arbitraje, no podemos negar que este remedio no ha sido utilizado de forma efectiva. La parte ganadora de un arbitraje suele concentrarse en los extremos del laudo que se pronuncian con respecto a las pretensiones principales y suele obviar reiteradamente la determinación de otros conceptos importantes como las costas y/o los intereses.

NULLA EXECUTIO SINE TITULO: EL ESCRUTINIO DE LOS LAUDOS EN LA PRÁCTICA DEL ARBITRAJE INTERNACIONAL COMO

MECANISMO

PARA OPTIMIZAR LA EJECUCIÓN DE LOS LAUDOS EN EL PERÚ

NULLA EXECUTIO SINE TITULO:THE SCRUTINY OF AWARDS IN THE PRACTICE OF INTERNATIONAL ARBITRATION AS A MECHANISM TO OPTIMIZE THE ENFORCEMENT OF AWARDS IN PERU

$14 \mathrm{En}$ el mismo orden de ideas, el Tribunal Constitucional español ha señalado que «el derecho a que se ejecuten los fallos judiciales se satisface cuando el juzgador adopta las medidas oportunas para llevar a efecto esa ejecución con independencia de la celeridad temporal en que las dicte; es la eficacia, y no el tiempo, la que sirve de pauta para determinar en cada caso los márgenes constitucionales de una pretendida vulneración de ese derecho. Por el contrario, cuando las medidas se adoptan por el órgano judicial con una tardanza excesiva e irrazonable, al margen de su eficacia objetiva, el derecho a un proceso sin dilaciones indebidas será el eventualmente conculcado» (doctrina vertida en las SSTC 26/1983, de 13 de abril y 26/1986, de 20 de febrero). Sobre este punto, el profesor Priori ha señalado que «el derecho a la efectividad de las resoluciones es el derecho que tienen las partes a que lo decidido por el órgano jurisdiccional sea cumplido. Para ello, se debe proveer al ciudadano de todos los medios adecuados para que se garantice la efectividad de las resoluciones judiciales» (Priori, 2003, p, 291). En la misma línea de ideas, en otra simbólica sentencia, el Tribunal Constitucional español expresó textualmente que «el derecho a que se ejecuten los fallos judiciales que reconocen derechos propios sólo se satisface cuando el órgano judicial adopta las medidas oportunas para llevar a efecto esa ejecución, con independencia de cuál sea el momento en que las dicta. Si esas medidas se adoptan, el derecho a la tutela judicial efectiva se habrá satisfecho, aunque si se adoptan con una tardanza excesiva e irrazonable puede considerarse lesionado el derecho al proceso sin dilaciones indebidas. Cuando, por el contrario, se adoptan, aunque sea con la mayor celeridad, medidas que no son eficaces para asegurar la ejecución o que, aun siendo en principio adecuadas quedan privadas de eficacia por no ir seguidas de las destinadas a complementarlas, no cabrá hablar seguramente de dilaciones indebidas" (María Luisa Tárraga y otros c. Consejo Superior de Investigaciones Científicas, fundamento 3). 
Sin embargo, lo más grave y cierto es que, sin parámetros, el juez de la ejecución del laudo estará atado de manos, como hemos ya visto. En efecto, no puede «arbitrar» y fijar estos parámetros que un tribunal arbitral no ha fijado, pues el juez de ejecución de laudo no tiene jurisdicción arbitral. Por ende, el juez de ejecución no podrá cumplir la misión constitucional que le ha sido encargada: ejecutar o hacer cumplir la efectividad de las decisiones (de cuarto grado, en los términos de Chamorro).

Por lo demás, muchos tribunales arbitrales optan por considerar ya resuelto el tema con solo indicar que «hay condena a intereses» o que «hay condena a costos», sin fijar los parámetros que permitan liquidar el monto. Si no hay parámetros, no estamos dentro del ámbito de lo «liquidable mediante simple operación aritmética» por el juez de ejecución. Y, como dichos tribunales arbitrales consideran que ya se pronunciaron sobre todos los puntos controvertidos dando respuesta a las pretensiones, rechazan el pedido de integración de laudo señalando que no hay nada que integrar. Esta concepción errónea de la misión arbitral es, sin duda, una afectación del derecho a la efectividad de segundo grado, en los términos ya definidos y explicados antes.

Se genera entonces un limbo para el justiciable y un círculo vicioso. El árbitro rechaza el pedido de integración de laudo porque lo considera completo, aunque sea obvio que faltan parámetros para convertir el mandato en uno liquidable y, por lo tanto, líquido en ejecución. Y el juez no puede fijar los parámetros que el tribunal arbitral omitió porque no tiene jurisdicción arbitral. Por lo tanto, el juez no puede ejecutar.

En nuestra opinión, este limbo en el cual quedan colocados algunos justiciables puede ser resuelto para el caso de los arbitrajes institucionales. Así, una de las soluciones que maximiza tanto la autonomía privada de las partes como la efectividad de las decisiones jurisdiccionales y evita el limbo antes descrito es que los centros de arbitraje peruanos incorporen dentro de sus reglamentos arbitrales el escrutinio de los laudos.

\footnotetext{
VIII. ¿QUÉ ES EL ESCRUTINIO DE LOS LAUDOS?

El escrutinio de los laudos es un mecanismo a través del cual una institución arbitral busca que el laudo sea de la mejor calidad posible, incrementando así las posibilidades de que sea ejecutado de forma efectiva. Asimismo, busca que el laudo esté debidamente motivado, incrementando asílas posibilidades de que sea cumplido voluntariamente por la parte perdedora. Por último, y como derivación de lo anterior, busca mitigar el riesgo de que el laudo sea anulado (Bond \& Paralika, 2015, p. 421).
} 
$\mathrm{Al}$ respecto, Bond y Paralika han señalado expresamente lo siguiente:

The scrutiny of awards pursuant to art. 33 is one of the most distinctive features of ICC arbitration. No other major arbitral institution has historically had a similar process. Art. 33 has two goals. First, it seeks to ensure that ICC awards are of as high a quality as possible with respect to the form of the award. Ensuring the formal quality of awards increases the likelihood that the award will be enforced. Second, it seeks to ensure that the award is well-reasoned and persuasive so as to increase the likelihood that it is complied with voluntarily by the losing party and is less susceptible to being set aside or denied enforcement (2015, p. 421).

Por otro lado, el profesor Gerbay se ha pronunciado de la siguiente manera: «In addition and more importantly perhaps, it is a last-minute opportunity for the institution to cast an eye on the award before it is issued to the parties. In other words, a requirement that any award be issued by the institution fulfills a "quality control" purpose» (Gerbay, 2016, p. 55) ${ }^{15}$.

Tal y como señala el profesor Gerbay, el escrutinio es una oportunidad de último minuto para que una institución ajena a las actuaciones del arbitraje revise el laudo antes de que este sea notificado a las partes. De alguna u otra manera, el escrutinio de los laudos es una especie de control de calidad. Así, los profesores Blackaby y Partasides han señalado que el escrutinio «helps to ensure a measure of "quality control", but the scrutiny is principally as to form and does not affect the tribunal's liberty of decision» (Blackaby, Partasides, Redfern \& Hunter, 2015, p. 604). En el mismo sentido, el profesor Born ha señalado que «experience teaches even the most self-confident tribunal that another set of eyes can be helpful in catching mistakes» (Born, 2015, p. 311).

El reglamento de arbitraje más emblemático que reconoce el escrutinio de los laudos es el Reglamento de Arbitraje de la Corte Internacional de Arbitraje de la Cámara de Comercio Internacional (en adelante, Reglamento de la CCI $)^{16}$. Al respecto, el artículo 33 del Reglamento de la CCI señala expresamente lo siguiente:

Artículo 33.- Examen previo del laudo por la Corte.- Antes de firmar un laudo, el tribunal arbitral deberá someterlo, en forma de proyecto,

$15 \mathrm{En}$ el mismo sentido, el profesor Verbist ha señalado expresamente que «the scrutiny of draft awards is an important and attractive feature of ICC arbitration as it generally improves their quality and enhances the enforceability of the award. Scrutiny is a fundamental feature of ICC arbitration and it increases confidence in the ICC arbitral process. It distinguishes ICC arbitration from proceedings conducted under other major international arbitration rules such as those of UNCITRAL or ICSID, which do not contain any equivalent provisions» (Verbist, Schäfer \& Imhoos, 2015, pp. 421-431).

16 Como veremos en la sección XII, existen numerosas instituciones arbitrales que reconocen en sus reglamentos el escrutinio de los laudos.

NULLA EXECUTIO SINE TITULO: EL ESCRUTINIO DE LOS LAUDOS EN LA PRÁCTICA DEL ARBITRAJE INTERNACIONAL COMO

MECANISMO

PARA OPTIMIZAR LA EJECUCIÓN DE LOS LAUDOS EN EL PERÚ

NULLA EXECUTIO SINE TITULO: THE SCRUTINY OF AWARDS IN THE PRACTICE OF INTERNATIONAL ARBITRATION AS A MECHANISM TO OPTIMIZE THE ENFORCEMENT OF AWARDS IN PERU 
a la Corte. Esta podrá ordenar modificaciones de forma y, respetando la libertad de decisión del tribunal arbitral, podrá llamar su atención sobre puntos relacionados con el fondo de la controversia. Ningún laudo podrá ser dictado por el tribunal arbitral antes de haber sido aprobado, en cuanto a su forma, por la Corte $^{17}$.

Como se puede apreciar, el artículo 33 del Reglamento de la CCI diferencia las modificaciones de forma de las modificaciones de fondo. La distinción es muy significativa. La Corte puede exigir modificaciones de forma, pero únicamente puede llamar la atención del tribunal arbitral con respecto a puntos de fondo. En relación con el escrutinio de fondo, Bond y Paralika han señalado expresamente lo siguiente:

The ICC Court does not act as an appellate court or verify that the legal and factual matters set out in the draft award are «correct». It can,

17 Al respecto, en el Statistical Report de la Cámara de Comercio Internacional «in 2012, only 2\% of all awards issued at the ICC were not the object of any suggestions of modification» (International Chamber of Commerce, 2013, p. 15). Asimismo, en el período 2008-2012, el profesor Greenberg ha señalado que «the ICC Court approved a total of 1,809 draft awards and decided not to approve 139 awards (for a total number of awards of 1,948 awards), which means that a "not insignificant" $7 \%$ of awards failed to meet the approval of the Court» (Greenberg, 2013, p. 89). Por otro lado, de acuerdo con las estadísticas de la CCl, «in 2013, no less than 471 awards were approved by the Court. These comprised 327 final awards, 104 partial or interim awards, and 40 awards by consent» (International Chamber of Commerce, 2014, p. 14). «In 2012, the Court approved 491 awards, comprising 341 final awards, 119 partial or interim awards and 31 awards by consent» (International Chamber of Commerce, 2013, p. 15). «In 2011, a total of 508 awards were approved by the Court, comprising 347 final awards, 120 partial or interim awards, and 41 awards by consent» (International Chamber of Commerce, 2012, p. 15). «In 2010, the Court approved 479 awards, comprising 305 final awards, 130 partial or interim awards, and 44 awards by consent» (International Chamber of Commerce, 2011 , p. 15). «In 2009, the total number of awards approved by the Court was 415 , comprising 265 final awards, 111 partial or interim awards, and 39 awards by consent» (International Chamber of Commerce, 2010, p. 14). «In 2008, 407 awards were approved, namely 261 final awards, 109 partial or interim awards, and 37 awards by consent» (International Chamber of Commerce, 2009, p. 14). Finalmente, el profesor Verbist ha explicado que «according to the statistics published by the Court, the latter gave an unqualified approval to only 5 draft awards in 2013. The remaining 466 awards were approved subject to modifications of form or with the attention of the arbitrators being drawn to points of substance, in order to enhance the quality and enforceability of the award. 40 draft awards were not approved, but sent back to the arbitral tribunal to be reworked and resubmitted (see "2013 Statistical Report" published in ICC International Court of Arbitration Bulletin, 25(1), 2014, p. 14). In 2012, the Court required modifications as to form and drew the attention of the arbitral tribunal to points of substance when approving $483(98 \%)$ awards. On a further 59 occasions, the Court requested that the arbitral resubmit its award for approval (see «2012 Statistical Report» published in ICC International Court of Arbitration Bulletin, 24(1), 2013, p. 15). In 2011, the Court used its power to require modifications as to form and draw the attention of the arbitral tribunal to points of substance when scrutinizing $496(97.6 \%)$ of the awards. On a further 37 occasions, the Court requested that the arbitral tribunal resubmit its award for approval (see «2011 Statistical Report» published in ICC International Court of Arbitration Bulletin, 23(1), 2012, p. 15). In 2010, the Court used its power to require modifications as to form and draw the attention of the arbitral tribunal to points of substance when scrutinizing $444(92.7 \%)$ of the awards. On a further 43 occasions, the Court requested that the arbitral tribunal resubmit its award for approval (see «2010 Statistical Report» published in ICC International Court of Arbitration Bulletin, 22(1), 2011, p. 15). In 2009, the Court required modifications as to form and/or drew attention to points of substance when approving 382 awards, leaving 33 awards approved without commentary by the Court. On a further 34 occasions, the Court requested that the arbitral tribunal resubmit its award for approval (see «2009 Statistical Report» published in ICC International Court of Arbitration Bulletin, 21(1), 2010, p. 14). In 2008, the Court used its power to require modifications as to form and draw the attention of the arbitral tribunal to points of substance when scrutinizing 363 of the awards, leaving 44 awards approved without commentary by the Court. On a further 25 occasions, the Court requested that the arbitral tribunal resubmit its award for approval (see «2008 Statistical Report» published in ICC International Court of Arbitration Bulletin, 20(1), 2009, p. 14) (Verbist et al., 2015, pp. 182-183). 
however, draw the arbitrators' attention to points of substance, without affecting their liberty of decision. The ICC Court exercises this right sparingly. It does not want to be seen as interfering inappropriately in the arbitrators' deliberations. It will generally only raise questions with regard to points of substance when the award's reasoning is internally inconsistent, illogical or incomplete, or points made in a dissenting opinion are not dealt with in the draft majority award. Its goal is not to affect the outcome of the award, but to ensure that the reasoning is clear and persuasive (Bond \& Paralika, 2015, p. 423).

El procedimiento de escrutinio se inicia cuando el tribunal arbitral le remite a la Secretaría Internacional de Arbitraje de la Cámara de Comercio Internacional (en adelante, Secretaría de la CCI) el proyecto de laudo sin firmar. El consejero del equipo al que se le ha asignado el caso será el primero en revisar el borrador del laudo. El consejero puede realizar comentarios al tribunal arbitral antes de que el laudo se someta a la Corte Internacional de Arbitraje de la Cámara de Comercio Internacional (en adelante, Corte de la CCI), si es que contiene problemas sustanciales de forma. Este podría ser el caso, por ejemplo, si es que el árbitro que redactó el borrador de laudo ha sido nombrado como árbitro por primera vez (Bond \& Paralika, 2015, p. 421).

Después de la revisión del consejero, el borrador es revisado también por el Secretario General, el Secretario General Adjunto o el Consejero General. Este segundo encargado de la revisión discute luego el laudo con el consejero. El consejero elabora a continuación un reporte del laudo a efectos de enviarlo a la Corte de la CCI. Los laudos son generalmente considerados en una sesión de comité. Sin embargo, si un laudo se deriva de asuntos particularmente complejos o novedosos, involucra a una parte estatal, llega a una decisión sobre un monto significativo en disputa o está acompañado de una opinión disidente, generalmente se someterá a una sesión plenaria. Si es que el proyecto de laudo se somete a una sesión plenaria, uno de los miembros de la Corte elaborará un reporte del laudo indicando si es que debería ser aprobado o enviado de vuelta al tribunal arbitral. La Corte de la CCI solo tiene en frente suyo el proyecto de laudo, los términos de referencia y otros laudos antes dictados en el arbitraje, junto con los comentarios de la Secretaría y el reporte de los miembros de la Corte. Ni la Corte de la CCI ni la Secretaría de la CCI revisan las actuaciones en el arbitraje como parte del procedimiento de escrutinio (Bond \& Paralika, 2015, p. 421).

Después de que ha discutido el laudo, la Corte de la CCI generalmente adoptará una de tres posibles decisiones. Primero, podría aprobar el proyecto de laudo tal y como ha sido presentado. Esto es realmente inusual porque incluso los mejores proyectos de laudo usualmente

NULLA EXECUTIO

SINE TITULO: EL

ESCRUTINIO DE

LOS LAUDOS EN

LA PRÁCTICA

DEL ARBITRAJE

INTERNACIONAL

COMO

MECANISMO

PARA OPTIMIZAR

LA EJECUCIÓN DE

LOS LAUDOS EN

EL PERÚ

NULLA EXECUTIO

SINE TITULO: THE

SCRUTINY OF

AWARDS IN THE

PRACTICE OF

INTERNATIONAL

ARBITRATION AS

A MECHANISM

TO OPTIMIZE THE

ENFORCEMENT

OF AWARDS IN

PERU 
contienen pequeños errores de forma (errores tipográficos o errores matemáticos, por ejemplo). Segundo, podría aprobar el borrador sujeto a algunas modificaciones. Esta es la decisión más común de la Corte de la CCI. La decisión de la Corte de la CCI es notificada al tribunal arbitral, el cual es invitado a presentar el laudo firmado a la Secretaría después de haber incorporado las modificaciones sugeridas. Cuando la Secretaría recibe el laudo firmado, es responsable de verificar que las modificaciones solicitadas por la Corte de la CCI hayan sido implementadas correctamente. Si es que las modificaciones sugeridas por la Corte de la CCI no han sido incorporadas de forma satisfactoria, la Secretaría puede derivar el laudo a la Corte para una nueva revisión. Tercero, la Corte de la CCI puede decidir no aprobar el proyecto de laudo e invitar al tribunal arbitral a enviarlo nuevamente, siempre que se tome en cuenta algunos comentarios. La Corte de la CCI adopta esta decisión cuando el proyecto de laudo tiene serios problemas de forma o sustancia. El tribunal arbitral puede reenviar nuevamente el laudo después de haber tomado en consideración los comentarios de la Corte de la CCI y el procedimiento de escrutinio es repetido (Bond \& Paralika, 2015, p. 422).

Los profesores Verbist y Schäfer explican el procedimiento de escrutinio de la siguiente manera:

When an ICC arbitral tribunal submits its draft award to the ICC for approval, the Counsel in charge of the case at the Secretariat of the Court will review the draft award and may offer comments or observations. Once the draft award has been revised in light of any comments the Counsel may have made, it is submitted to the Court together with the Terms of Reference. A Court member is appointed as rapporteur to submit a report to the Court, which then deliberates and decides whether or not to approve the award.

The Court may (i) approve the draft award, (ii) approve the draft award but invite the arbitral tribunal to make changes to it when finalizing it for notification to the parties, or (iii) invite the arbitral tribunal to make changes to the draft award and to submit a revised version to a future session.

Pursuant to Article 33 of the ICC Rules, the award must be approved as to form. Requirements of form include, for example, whether reasons have been provided, whether the arbitral tribunal has dealt with all of the issues submitted to it, and whether formal requirements at the place of the arbitration have been met. However, Article 33 also permits the Court to make comments on the substance «without affecting the arbitral tribunal's liberty of decision». Examples include problems of computation, contradictory findings of fact or law, decisions made 
ultra petita, and failure to apply or make reference to the applicable law (Verbist et al., 2015, p. 421).

El procedimiento de escrutinio puede ser resumido sucintamente con el siguiente gráfico ${ }^{18}$ :

NULLA EXECUTIO

SINE TITULO: EL

ESCRUTINIO DE

LOS LAUDOS EN

El Tribunal Arbitral redacta un proyecto de

borrador de laudo

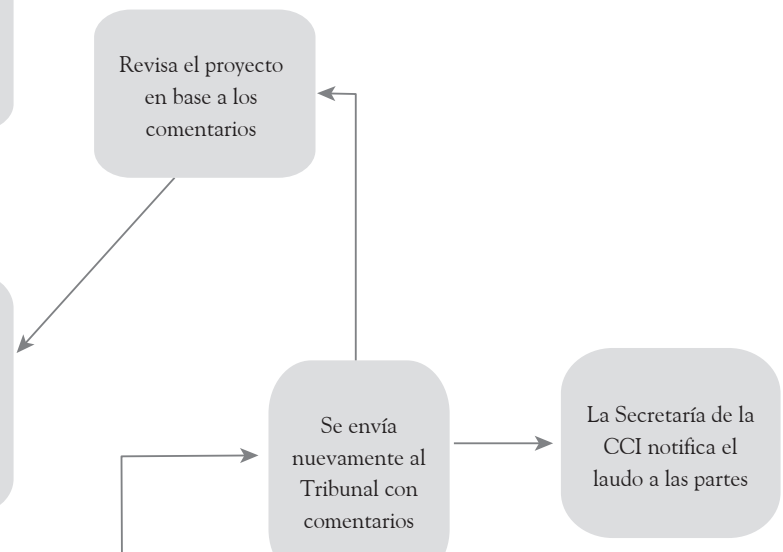

El Tribunal Arbitral le remite el proyecto de laudo a la Secretaría de la CCI

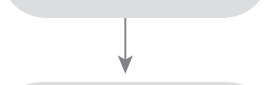
comentarios

LA PRÁCTICA

DEL ARBITRAJE

INTERNACIONAL COMO

MECANISMO

PARA OPTIMIZAR

LA EJECUCIÓN DE

LOS LAUDOS EN

EL PERÚ

NULLA EXECUTIO

SINE TITULO: THE

SCRUTINY OF

AWARDS IN THE

PRACTICE OF

INTERNATIONAL

El Consejero del equipo que está a cargo del caso revisa el proyecto del laudo y luego un staff privado

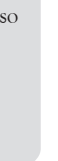
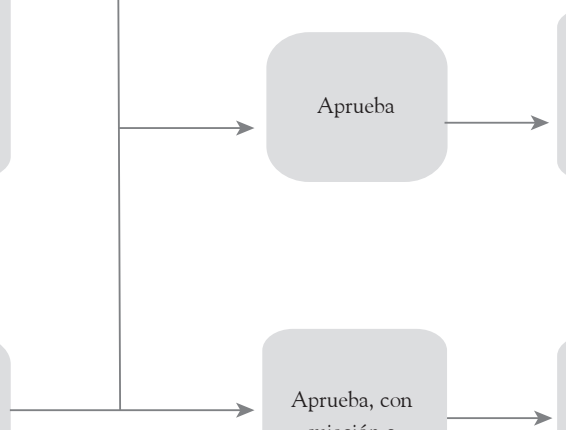

El Tribunal Arbitral firma el laudo y lo envía a la Secretaría ARBITRATION AS

A MECHANISM

TO OPTIMIZE THE ENFORCEMENT OF AWARDS IN PERU

La Corte de la CCI realiza el escrutinio del proyecto de laudo

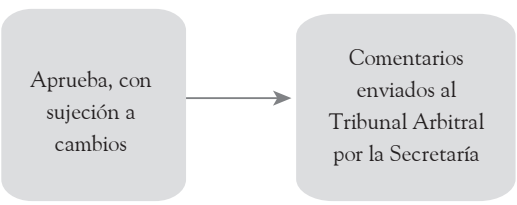

Tal y como señala el profesor Carreteiro, «the award receives the ICC stamp only after scrutiny» (2016, p. 208). En efecto, lo importante es tener en cuenta que antes de llevar a cabo el escrutinio el proyecto

18 La información del presente gráfico ha sido extraída de la presentación de J osé Ricardo Feris (2015). Sobre este particular, tal y como señala el profesor Verbist, «all draft awards undergo a three-step review process, starting with the counsel of the team in charge of the arbitration that has followed the proceedings since the inception of the arbitration, followed by review by myself, the Deputy Secretary General, the General Counsel, or the Managing Counsel, before being submitted for the Court's scrutiny. For certain arbitrations, generally those involving state parties or dissenting opinions, a Court member will draft a report with recommendations on the draft award» (Verbist et al., 2015, pp. 334-335). 
de laudo no puede ser considerado como un laudo, por más que se encuentre perfectamente bien redactado y motivado. En ese sentido, el profesor Loquin ha señalado expresamente que «prior to scrutiny a draft award is not an award under the rules» (p. 428; en el mismo sentido, véase también Born, 2012, p. 2352).

\section{IX.EL ESCRUTINIO DE LOS LAUDOS COMO MECANISMO QUE MAXIMIZA LA AUTONOMÍA PRIVADA Y LA EFECTIVIDAD DE LAS DECISIONES JURISDICCIONALES}

El escrutinio de los laudos salvaguarda la autonomía privada porque son las partes quienes voluntariamente deciden incorporar a su contrato un convenio arbitral, sometiéndose al Reglamento de la CCI que prevé, en el artículo 33, el escrutinio de los laudos. En otras palabras, el escrutinio no es impuesto a las partes, sino que son estas quienes contractualmente deciden que la corte de arbitraje que administraría el arbitraje intervenga antes de que se notifique el laudo a las partes, con el fin de mitigar el riesgo de anulación y garantizar la ejecutabilidad del laudo. Al tratarse de un pacto libre de las partes, no se restringe la autonomía privada sino que, muy por el contrario, se la salvaguarda.

Sobre el particular, el profesor Gerbay ha señalado expresamente lo siguiente: «The mission of supervision conferred to the institution is therefore purely contractual». El escrutinio de los laudos, por otra parte, salvaguarda también la efectividad de las decisiones jurisdiccionales, puestoque, precisamente, tiene como función incentivar el cumplimiento voluntario de la parte perdedora, garantizar la ejecutabilidad del laudo y mitigar el riesgo de que sea anulado. Así lo ha reconocido la doctrina en diversas fuentes y la jurisprudencia internacional, la que procedemos a compartir a continuación:

En el caso Cubic Defense System, la Corte de Casación señaló expresamente lo siguiente: «In this respect the Court of Appeal has correctly judged that the scrutiny of draft awards implies no undue interference in the jurisdictional mission of the arbitrator, but merely aims at guaranteeing the effectiveness of the arbitration proceedings» (véase Société Cubic Defense Systems Inc. c. Chambre de Commerce Internationale, Cour d'Appel; Société Cubic Defense Systems Inc. c. Chambre de Commerce Internationale, Tribunal de Grande Instance de Paris; Société Cubic Defense Systems Inc. c. Chambre de Commerce Internationale, Cour de Cassation; las cursivas son nuestras).

Por otra parte, el profesor Gerbay se ha pronunciado de la siguiente manera: 
At the end of an arbitration, in the absence of amicable settlement, the tribunal issues an award. In order to ensure that the award adequately deals with the parties' claims and to improve its enforceability, a number of arbitration rules require the tribunal to provide the institution's secretariat with a copy of the award in draft form for review. This review is what is referred to as «scrutiny» of the award (Gerbay, 2016, p. 150; las cursivas son nuestras).

De igual manera, la profesora Leoveanu ha señalado expresamente lo siguiente:

The Court's scrutiny is one of the key aspects of ICC arbitration. Article 33 is one of the essential provisions of the ICC Rules from which the parties cannot derogate. The purpose of the scrutiny is to maximize the legal effectiveness of an award by identifying any defects that could be used in an attempt to have it set aside at the place of the arbitration or resist its enforcement elsewhere (Leoveanu, 2016, p. 349); las cursivas son nuestras).

Finalmente, el profesor Zahariev ha señalado expresamente lo siguiente:

One of the main and well-known advantages of arbitration is the speed of the proceedings. In order to enhance their timeliness and efficiency, the Rules of some arbitral institutions (e.g. Art. 33 of the ICC Rules) provide for the so called «scrutiny of the award» - a procedure of formal examination of the draft award carried out before it is signed by the arbitrators - and the parties are notified of it. The idea behind such procedure is to maximize the legal effectiveness of an award by identifying any defects that could be used in an attempt to have it set aside at the place of the arbitration or resist its enforcement elsewhere (2015; las cursivas son nuestras).

Como se puede apreciar, la jurisprudencia y la doctrina internacional son unánimes al señalar que la función del escrutinio es garantizar la efectividad de los laudos en virtud del pacto de las partes. En ese sentido, el escrutinio es un mecanismo que resguarda los dos derechos fundamentales que se encontraban en conflicto, puesto que no sacrifica ni la autonomía privada de las partes ni la efectividad de las decisiones jurisdiccionales, sino que, muy por el contrario, las maximiza.

El escrutinio de los laudos, además, es especialmente relevante para ejemplos como los que fueron explicados anteriormente, en los que los laudos sufrían una serie de tropiezos. Como señalamos, los principales tropiezos en la ejecución de los laudos en el Perú radican en la falta de determinación de las costas y/o los intereses, problemas matemáticos o de cálculos y motivaciones insuficientes. Lo cierto es que el escrutinio de los laudos es el mecanismo por antonomasia para resolver esos problemas.

NULLA EXECUTIO

SINE TITULO: EL

ESCRUTINIO DE

LOS LAUDOS EN

LA PRÁCTICA

DEL ARBITRAJE

INTERNACIONAL

COMO

MECANISMO

PARA OPTIMIZAR

LA EJECUCIÓN DE

LOS LAUDOS EN

EL PERÚ

NULLA EXECUTIO

SINE TITULO: THE

SCRUTINY OF

AWARDS IN THE

PRACTICE OF

INTERNATIONAL

ARBITRATION AS

A MECHANISM

TO OPTIMIZE THE

ENFORCEMENT

OF AWARDS IN

PERU 
Tal y como ha sido reconocido por variada doctrina internacional, el escrutinio de los laudos sirve, por ejemplo, para que se determinen correctamente las costas y/o los intereses, se corrijan los problemas matemáticos y/o de cálculos y para garantizar que la motivación de los laudos sea adecuada.

Sobre este particular, por ejemplo, el profesor Appel ha señalado expresamente lo siguiente: «The most typical issues identified on review concern the calculation of costs. Senior staffs also satisfy themselves that the requirements of the New York Convention have been met and that all claims are addressed» (2012, p. 84).

De igual manera, los profesores Blackaby y Partasides se han pronunciado en los siguientes términos: «The ICC prides itself on the overall quality of ICC awards and the scrutiny process acts as a measure of quality control, ensuring, amongst other things, that the arbitrators deal with all of the claims. These include interest and costs, with which the arbitrators are called upon to deal» (Blackaby et al., 2015, p. 501).

Finalmente, el profesor Verbist ha señalado expresamente lo siguiente:

The Court's scrutiny of the draft arbitral award (Article 33) is intended to avoid that any such typographical or computational errors or errors of similar nature are contained in the award. If such errors are nevertheless found after the award has been notified to the parties, the question therefore arises as to whether the parties may turn again to the same arbitral tribunal or whether new proceedings must be commenced Verbist et al., 2015, pp. 190-191).

Como se puede apreciar, el escrutinio de los laudos tiene especial relevancia para la determinación de los costos y los intereses, conceptos que normalmente generan muchos problemas cuando los laudos son ejecutados ante el Poder Judicial. Una de las prácticas más interesantes que se utiliza para garantizar la ejecutabilidad de estos conceptos es la práctica adoptada por la CCI. En efecto, de acuerdo al Reglamento de la CCI, desde el momento en que la Secretaría de la CCI le transmite el asunto al tribunal arbitral, elabora un documento denominado «ICC Award Checklist», el cual tiene como propósito proporcionar a los árbitros una guía en el momento en que redactan sus laudos. Este no constituye un documento exhaustivo y vinculante. Simplemente busca facilitar la tarea al tribunal en el momento en que redacta sus laudos (Verbist et al., 2015, pp. 182-183).

Este «checklist» provee una serie de puntos formales que todos los árbitros deben considerar al momento de redactar el laudo. Incluye siempre una serie de requerimientos generales, tales como la necesidad de mencionar el caso CCI de referencia, así como la necesidad de la identificación de las 
partes y sus representantes, los árbitros y el convenio arbitral. Además, el «checklist» recomienda incluir en el laudo los siguientes puntos: los antecedentes del procedimiento arbitral con un resumen de cada uno de los pasos procesales, así como las decisiones adoptadas por la Corte de la CCI con respecto al procedimiento arbitral, incluyendo el tiempo límite para dictar el laudo y cualquier extensión otorgada por la Corte de la CCI. El «checklist» también establece que el laudo debe incluir una decisión con respecto a los costos del arbitraje y su distribución entre las partes. Finalmente, también provee recomendaciones prácticas para redactar la sección dispositiva del laudo y, en particular, la última página para la firma de los árbitros (Verbist et al., 2015, pp. 182-183).

Así, cuando la Secretaría de la CCI recibe un laudo para realizar el escrutinio, lo primero que hace es revisar si es que el tribunal arbitral se ha pronunciado sobre todos los ítems del «checklist». Si alguno de estos ítems no ha sido abordado en el laudo, la Secretaría de la CCI reenviará el «checklist» al tribunal arbitral, con el fin de que identifique los puntos faltantes (Verbist et al., 2015, pp. 182-183). Este «checklist» constituye una práctica que debería ser adoptada por todas las instituciones arbitrales del mundo porque es de mucha utilidad para facilitar la ejecución del laudo.

\section{UNA PERSPECTIVA PRÁCTICA: ¿POR QUÉ EL} ESCRUTINIO DE LOS LAUDOS ES NECESARIO?

De acuerdo con el profesor Feris (2015), las cuestiones que son más comúnmente abordadas por la CCI son las siguientes:

- parte dispositiva

NULLA EXECUTIO

SINE TITULO: EL

ESCRUTINIO DE

LOS LAUDOS EN

LA PRÁCTICA

DEL ARBITRAJE

INTERNACIONAL

COMO

MECANISMO

PARA OPTIMIZAR

LA EJECUCIÓN DE

LOS LAUDOS EN

EL PERÚ

NULLA EXECUTIO

SINE TITULO: THE

SCRUTINY OF

AWARDS IN THE

PRACTICE OF

INTERNATIONAL

ARBITRATION AS

A MECHANISM

TO OPTIMIZE THE

ENFORCEMENT

OF AWARDS IN

PERU

- consistencia y suficiencia de motivación

- cuestiones con la norma aplicable

- intereses y costos

- debido proceso

- cálculos

- laudos finales que no son ejecutables

Pues bien, a efectos de poner en evidencia por qué el escrutinio de los laudos es indispensable en los distintos reglamentos de arbitraje peruanos, resulta pertinente citar algunos ejemplos de casos CCI (información extraída de Feris, 2015):

1. Decisión sobre el interés sin indicación de la tasa de interés y su fecha de inicio: 
That Claimant compensates Respondent in the amount of US\$ XX in respect of the breaches under the Service Agreement and US\$ XX in respect of the breaches under the Maintenance Agreement, in each case together with interest thereon from the date of maturity through the date of payment.

2. Discrepancia entre el cuerpo y la parte dispositiva del laudo:

Added together, Respondent owes Claimant the total amount of EUR 3'115'241.23. Respondent shall pay to Claimant the amount of EUR 3'113'231.23.

3. La cuantificación de los montos se deja para la ejecución judicial del laudo:

Claimant shall reimburse Respondent for the amounts disbursed in connection with the works related to the environmental repair of the Building. To that amount, monthly interest of $1 \%$ (not compounded) shall apply as well as the contractual penalty of $5 \%$. The exact quantification of those amounts shall be made on the basis of the invoices and relevant documents that Respondent shall produce, which will also include the necessary calculations. In the event Claimant objects to those amounts or calculations, or to the documents produced, the quantification shall be made during the judicial enforcement of the award.

4. La cuantificación de los montos se deja para la ejecución judicial del laudo:

The Tribunal orders XX to pay YY, an amount to be assessed upon the enforcement of the present award, and determined in accordance with 23.1 above, with the figure reached in said calculation to be deducted from the amount referred to in 23.2.

Financial assessment upon enforcement is rendered necessary because the entitlement being invoked by the plaintiff $\mathrm{XX}$ is contentious -i.e. both its existence and its «quantum» will depend upon what is ultimately decided in an ordinary court of law, which in this instance is the ZZ Administrative and Tax Court - and accordingly no exact and defined value can presently be established in this arbitral award.

No cabe la menor duda, pues, de que es indispensable que se incluya el escrutinio de los laudos en los distintos reglamentos de arbitraje peruanos, con el fin de evitar que se presenten en la realidad casos tan absurdos como los citados. En definitiva, la incorporación de este mecanismo en los reglamentos permitirá garantizar la efectividad de las decisiones jurisdiccionales. 


\section{XI.LAS CRÍTICAS INFUNDADAS AL ESCRUTINIO DE LOS LAUDOS}

Si bien la doctrina mayoritaria no duda de las virtudes que el escrutinio de los laudos provee a las partes de un arbitraje, no han faltado quienes han realizado numerosas críticas —en nuestra opinión, infundadasa este mecanismo. A continuación, compartiremos cuáles han sido las principales críticas y explicaremos porque cada una de ellas es equivocada:

\section{a. Se le atribuye una función jurisdiccional a una entidad administradora}

Hay quienes han señalado que el escrutinio de los laudos es un mecanismo inadmisible porque implicaría atribuirle función jurisdiccional a una entidad administradora. Ello es equivocado. Si bien la Corte de la CCI prevé el escrutinio de los laudos, el artículo 1, inciso 2 del Reglamento de la CCI es claro al señalar que «la Corte no resuelve por sí misma las controversias». Ella administra la resolución de controversias por tribunales arbitrales, de conformidad con el Reglamento de Arbitraje de la CCI. La Corte es el único órgano autorizado a administrar arbitrajes bajo el Reglamento, incluyendo el examen previo y la aprobación de laudos dictados de conformidad con el Reglamento.

$\mathrm{Al}$ respecto, el profesor Gerbay ha señalado que

the intervention of the organ of the institution cannot be qualified of jurisdictional. The arbitration rules of the institutions affirm this clearly. Article 2 of the ICC Rules provides that «The Court does not itself resolve disputes», Article 24-1 of the rules of arbitration of the Franco Arab Chamber of Commerce provide that the intervention of the Council in the scrutiny of draft awards shall not lead to it being «considered as exercising the role of arbitrator» (2016, p. 159).

Con respecto a la jurisprudencia internacional, existen reiterados casos que se han pronunciado al respecto. Desde el caso Cubic Defense System la jurisprudencia francesa se ha mantenido estable (véase Société Cubic Defense Systems Inc. c. Chambre de Commerce Internationale, Cour d'Appel; Société Cubic Defense Systems Inc. c. Chambre de Commerce Internationale, Tribunal de Grande Instance de Paris; Société Cubic Defense Systems Inc. c. Chambre de Commerce Internationale, Cour de Cassation). En 2002, en el caso Société Lear c. Chambre de Commerce Internationale, el Tribunal de Grande Instances de París reafirmó que el escrutinio de los laudos realizado por la CCI no constituía una función jurisdiccional. De igual manera, en los años 2007 y 2009, el Tribunal de Grande Instance y la Corte de Apelaciones de París, respectivamente, confirmaron en el caso Société SNF la validez de la dicotomía entre lo jurisdiccional y lo

NULLA EXECUTIO SINE TITULO: EL ESCRUTINIO DE LOS LAUDOS EN LA PRÁCTICA DEL ARBITRAJE INTERNACIONAL COMO

MECANISMO

PARA OPTIMIZAR LA EJECUCIÓN DE LOS LAUDOS EN EL PERÚ

NULLA EXECUTIO SINE TITULO:THE SCRUTINY OF AWARDS IN THE PRACTICE OF INTERNATIONAL ARBITRATION AS A MECHANISM TO OPTIMIZE THE ENFORCEMENT OF AWARDS IN PERU 
administrativo. Asimismo, ratificaron el hecho de que el escrutinio de los laudos no se enmarcaba en la función jurisdiccional que el Reglamento de la CCI reserva exclusivamente al tribunal arbitral (Société SNF SAS c Chambre de Commerce Internationale, Tribunal de Grande Instance; Société SNF SAS c Chambre de Commerce Internationale, Court d'appel). En 2009, en el caso Trioplast, la Cour de Cassation francesa confirmó nuevamente la dicotomía entre lo jurisdiccional y lo administrativo, sugiriendo que dicha dicotomía se encontraba basada en el Reglamento de la CCI ( $\mathrm{r} r \mathrm{X} v$ Trioplast $\mathrm{AB})$. Más adelante, en 2012, el Tribunal de Grande Instance de París confirmó la posición de que las actividades de la CCI (incluyendo las decisiones con respecto a recusaciones y a la constitución del tribunal arbitral) son administrativas por su naturaleza. Así, solamente las decisiones del tribunal arbitral pueden ser jurisdiccionales ${ }^{19}$.

Teniendo ello en cuenta, no cabe la menor duda de que, cuando las instituciones arbitrales realizan el escrutinio de los laudos, están ejerciendo una función administrativa.

\section{b. El escrutinio de la institución arbitral interfiere en la función jurisdiccional del tribunal arbitral}

El profesor Greenberg ha señalado que el «award scrutiny goes to the core of the question of the extent to which an administering institution can —or should — intervene with arbitral proceedings» (Greenberg, 2013, p. 89). En ese sentido, resulta pertinente analizar hasta qué punto el escrutinio de los laudos interfiere en la función jurisdiccional del tribunal arbitral.

Algunos autores han señalado que el escrutinio sí implica una interferencia en la función jurisdiccional del tribunal arbitral porque el impacto que tiene la revisión de la institución arbitral en el laudo es muy significativo. En ese sentido, señalan que la institución arbitral no debería tener la posibilidad de afectar el proceso de redacción de laudo. $\mathrm{Al}$ respecto, el profesor Fry se ha pronunciado de la siguiente manera: «The role of the ICC in scrutinizing draft awards goes as far

19 Sobre este particular, el profesor Gerbay ha señalado que «since the decision in Cubic Defense Systems, French "case law" on the matter has been remarkably stable. In 2002, in "Société Lear v. Chambre de Commerce Internationale", the courts reaffirmed the principle that the ICC's scrutiny of draft awards does not interfere with a so-called jurisdictional mission of the arbitrator. Likewise, in 2007 and 2009, the Tribunal de Grande Instance and the Court of Appeal of Paris respectively confirmed, in the Sociéte SNF case, both the validity of the "jurisdictional" v. "administrative" dichotomy, and the fact that the scrutiny provisions of the ICC rules do not encroach on the jurisdictional function which the ICC rules reserve to the arbitrators. In 2009, in the Trioplast case, the Cour de Cassation confirmed again the "jurisdictional" versus "administrative" dichotomy, in a manner which suggested that such a dichotomy was grounded on the ICC rules. In 2012, the Tribunal de Grande Instance de Paris confirmed the position that the activities of the ICC (including the decisions on challenges or the constitution of a tribunal) are administrative in nature, and that only those of the Tribunal may be jurisdictional» (Gerbay, 2016, p. 140). 
as identifying "flawed legal reasoning and procedural errors", which by definition means that, if the recommended corrections are accepted by the tribunal, they will impact the outcome of the case» (Fry, 2012, p. 14$)^{20}$.

De igual manera, el profesor Chen se ha pronunciado con las siguientes palabras: «In one extreme example, the case-handlers at the Beijing Arbitration Commission will point to the attention of the arbitrators' inconsistencies between the decision set out in the draft award and previous decisions rendered in similar cases, thereby "actually influencing decisions on the merits”»(Chen, 2007, p. 327).

Incluso han existido casos internacionales en que se ha argumentado que el escrutinio de los laudos era ilícito. Así, en el caso Cubic Defense System, seguido ante la Corte de Apelaciones francesa, Cubic argumentó que su contrato con la CCI era ilícito porque vulneraba el orden público internacional, en la medida en que el Reglamento de la CCI permitía una indebida interferencia de la institución arbitral en la misión del tribunal arbitral. La Corte de Apelaciones rechazó los argumentos de Cubic sobre la base de los siguientes argumentos:

the [lower judges] noted that the rules of arbitration of the ICC ensure the distinction between the function of organization of the arbitration, in particular through the «international court of arbitration», and the jurisdictional function, left entirely to the arbitrators, the «court» having no jurisdictional power at all. [...] in this respect the Court of Appeal has correctly judged that the scrutiny of draft awards implies no undue interference in the jurisdictional mission of the arbitrator, but merely aims at guaranteeing the effectiveness of the arbitration proceedings» (Société Cubic Defense Systems Inc. c. Chambre de Commerce Internationale, Cour d'Appel; las cursivas son nuestras; Société Cubic Defense Systems Inc. c. Chambre de Commerce Internationale, Tribunal de Grande Instance de Paris; Société Cubic Defense Systems Inc. c. Chambre de Commerce Internationale, Cour de Cassation).

En nuestra opinión, no es correcto afirmar que el escrutinio de los laudos interfiere en la función jurisdiccional, puesto que la institución arbitral únicamente puede ordenar modificaciones de forma y - respetando siempre la libertad de decisión del tribunal arbitralllamar su atención sobre aspectos relacionados con el fondo de la controversia, como señala el artículo 33 del Reglamento de la CCI.

20 De igual manera, el profesor Gerbay ha señalado expresamente que 'the impact of the institutions' review of draft awards is not insignificant (especially when scrutiny concerns the merits of the case). When the institution suggests or imposes a change (whether on form or substance) which then finds its way into the award, the institution effectively directly affects the drafting of the tribunal's decision [... ]. In short, the scrutiny of draft awards is a function which has the potential to affect the process significantly» (2016, p. 55).

NULLA EXECUTIO SINE TITULO: EL ESCRUTINIO DE LOS LAUDOS EN LA PRÁCTICA DEL ARBITRAJE INTERNACIONAL COMO

MECANISMO

PARA OPTIMIZAR LA EJECUCIÓN DE LOS LAUDOS EN EL PERÚ

NULLA EXECUTIO SINE TITULO:THE SCRUTINY OF AWARDS IN THE PRACTICE OF INTERNATIONAL ARBITRATION AS A MECHANISM TO OPTIMIZE THE ENFORCEMENT OF AWARDS IN PERU 
En ese sentido, a pesar de que la Corte de la CCI tenga la potestad de realizar un examen previo del laudo, el tribunal arbitral sigue teniendo independencia absoluta para emitir un fallo que se adecúe únicamente a su criterio. Las recomendaciones que realiza la Corte de la CCI con respecto al contenido del laudo no son vinculantes. Así, teniendo en cuenta que el tribunal arbitral tiene la potestad de rechazar cualquier recomendación de la Corte de la CCI con respecto a la sustancia del laudo, es evidente que el tribunal se mantiene siempre inmune a cualquier interferencia (Gerbay, 2016, p. 64).

Sobre este particular, el profesor Loquin ha señalado expresamente que «[t]he institution's organ does not intervene on the content of the award. Scrutiny is by nature incapable of reforming the decision» (1990, p. 434). En el mismo sentido, el jurista Zahariev ha señalado que «the scrutiny may result in underlining procedural or substantive legal matters, but without prejudice to the Arbitral Tribunal's decision-making power. Drawing the Arbitral Tribunal's attention to such matters, but letting the arbitrators render the final form of their award, is an important guarantee for the liberty of decision» (Zahariev, 2015; las cursivas son nuestras).

Asimismo, se debe tener en cuenta que el escrutinio es realizado por la Corte de la CCI cuando ya no existe ningún tipo de debate adversativo entre las partes. No solo la etapa de instrucción ya culminó, sino que incluso ya concluyeron todas las actuaciones arbitrales. Sobre este particular, el profesor Gerbay se ha pronunciado de la siguiente manera: «By nature, this question [scrutiny] escapes adversarial debates. It takes place after the debates, at a moment when they have been exhausted. The organ of the arbitral institution indeed intervenes during the deliberation phase, at a moment when no new applications may be made and no new arguments may be raised» (Gerbay, 2016, p. 150).

Es verdad que las recomendaciones de la Corte de la CCI pueden modificar significativamente la redacción del tribunal arbitral. Así lo ha resaltado el jurista Greenberg, señalando que «the Court's comments can result in significant changes to the award» (2013, p. 101). No obstante, lo cierto es que lo que no puede modificarse de ninguna manera es el criterio del tribunal arbitral, puesto que, de lo contrario, se vería seriamente infringida su libertad de decisión.

Algunos podrían afirmar que, si bien el escrutinio de los laudos es un mecanismo bienintencionado, puede ser utilizado de forma perversa. Así, podría ocurrir que la institución arbitral le indique al tribunal arbitral que el laudo no está bien motivado y le intente imponer ciertas modificaciones de fondo. En nuestra opinión, si ello sucede, el problema no es el escrutinio de los laudos como mecanismo, sino la forma en que 
es utilizado por la institución arbitral ${ }^{21}$. Por ello, estamos totalmente de acuerdo con el profesor Greenberg cuando señala que «[i]n assessing award scrutiny the real question is not what is contained in a brief provision like Article 33 of the ICC Rules, but how the provision is applied and how the process works internally» (Greenberg, 2013, p. 96).

Distinto es el caso de los temas de forma. En dichos casos, las sugerencias de la Corte de la CCI sí son vinculantes, por lo que el tribunal arbitral debe acatarlas si es que quiere que el laudo sea aprobado y, por ende, surta efectos en la realidad. Al respecto, el profesor Grigera ha señalado expresamente lo siguiente: «[m] odifications on the form are mandatory. If the tribunal refuses to include them in the award, the award will not be approved by the ICC Court and the Secretariat will therefore not communicate it to the parties» $(1999$, p. 70).

Es importante tener en cuenta qué debe entenderse por forma. Bajo el criterio del profesor Grigera, «form includes not only compliance with formal requirements, errors of a mathematical, computational or clerical nature, but also internal inconsistencies in the award, absence of reasons, or of adequate reasons, and decisions ultra or infra petita» (Grigera, 1999, p. 70). Hay quienes afirman que este ejercicio de la Corte de la CCI con respecto a los temas de forma es arbitrario porque el laudo debería ser intangible. Sin embargo, nosotros consideramos que están equivocados debido a que, con respecto a los temas de fondo, se preserva siempre la libertad de decisión del tribunal arbitral. Por otro lado, con respecto a los temas de forma, han sido las propias partes del arbitraje quienes, en virtud de su autonomía privada, han decidido libremente someterse a un reglamento arbitral que contempla el escrutinio de los laudos.

Es importante tener en cuenta que el escrutinio de los laudos es una de las provisiones del Reglamento de la CCI más esenciales y más conocidas, por lo que no cabe que las partes de un arbitraje aleguen que no sabían a lo

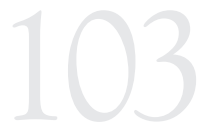

NULLA EXECUTIO SINE TITULO: EL ESCRUTINIO DE LOS LAUDOS EN LA PRÁCTICA DEL ARBITRAJE INTERNACIONAL COMO

MECANISMO

PARA OPTIMIZAR LA EJECUCIÓN DE LOS LAUDOS EN EL PERÚ

NULLA EXECUTIO SINE TITULO: THE SCRUTINY OF AWARDS IN THE PRACTICE OF INTERNATIONAL ARBITRATION AS A MECHANISM TO OPTIMIZE THE ENFORCEMENT OF AWARDS IN PERU

21 Asimismo, también es relevante tener en consideración el alcance del escrutinio que realizan las instituciones arbitrales. Al respecto, el profesor Gerbay ha señalado expresamente que «the extent of the review varies from one institution to the next. On one side of the spectrum one finds WIPO, which rules merely contemplate that the Tribunal may consult the Center with regard to matters of form, particularly to ensure the enforceability of the award. On the other side of the spectrum one finds the ICC which is empowered to impose modifications as to the form of the award, and also to suggest changes to its substance. At the ICC, the proportion of institutional awards modified as a result of scrutiny is not insignificant. In 2012, only $2 \%$ of all awards issued at the ICC were not the object of any suggestions of modifications. In between lies a number of institutions. Some, for instance, may make non-binding suggestions as to both form and merits (Article 28 SIAC rules (2013); Article 32.3 J AMS rules (2011)). Others may draw the tribunal's attention to any provision of the rules with which the draft award does not comply (Article 30.4 Milan rules (2010)). When institutional rules contain no express provisions as to scrutiny, the review process tends to be less extensive, with the institution often only drawing to the tribunal's attention the more important errors of form» (Gerbay, 2016, pp. 100-101). Asimismo, el profesor G reenberg señala que la $\mathrm{CCl}$ «usefully distinguishes between matters of form, matters that are "quasi substantive" and matters that are "usually substantive". He seems to indicate that, depending on the circumstances, such questions as "whether the reasoning in the award is consistent with the parties' pleadings or whether the rationale for the decisions could surprise the parties (iura novit curia)" or even "whether the reasoning is sufficient" would not necessarily be substantive» (Greenberg, 2013, p. 99). 
que se estaban sometiendo. Tan es así que, cuando las partes se someten al Reglamento de la CCI, una de las pocas provisiones inderogables es el escrutinio de los laudos. Sobre este particular, el profesor Verbist ha señalado expresamente lo siguiente: «In principle, there can be no doubt that the parties are not free to alter certain provisions containing essential features of the ICC arbitration procedure. Thus, the parties may not agree to exempt the arbitral tribunal from drawing up the Terms of Reference (Article 23) or submitting its award in draft form to the Court for scrutiny (Article 33)» (Verbist et al., 2015, p. 106) ${ }^{22}$.

De igual manera, los profesores Bond y Paralika han señalado expresamente lo siguiente: «The ICC Court will generally respect any agreement of the parties to modify the Rules. However if the parties' agreement to modify the ICC Rules relates to elements that the ICC Court considers to be mandatory, such as the establishment of the Terms of Reference or scrutiny of draft awards, the Court will refuse to administer the arbitration» (2015, p. 348).

Las partes podrían haberse sometido a otro reglamento arbitral; no obstante, al someterse al Reglamento de la CCI, han aceptado contractualmente someterse a todas sus disposiciones, incluido el escrutinio de los laudos. No cabe pues que, de manera absolutamente inconsecuente, afirmen luego que el escrutinio de los laudos interfiere en la función jurisdiccional del tribunal arbitral. Ello no implicaría más que ir en contra de sus propios actos.

\section{c. Demora innecesariamente el procedimiento arbitral}

Existen, además, numerosos autores que han criticado el escrutinio de los laudos afirmando que este demora innecesariamente el procedimiento arbitral. Al respecto, el profesor Verbist ha señalado que existen autores que han criticado el escrutinio de los laudos en los siguientes términos: «This rule has been criticized as it delays the proceedings and could be considered as an infringement of the arbitral tribunal's freedom of decision» (Verbist et al., 2015, pp. 181-182).

No obstante, se olvida que, en realidad, el escrutinio de los laudos es un mecanismo que contribuye a que el conflicto sea resuelto más rápidamente, puesto que su propósito último es facilitar la ejecución y evitar que el laudo sea anulado. En buena cuenta, se trata de un

22 De igual manera, el profesor Verbist ha señalado que "[a]ccording to Article 1 of Appendix I to the Rules, it is the ICC International Court of Arbitration that has the final word as to whether or not provisions departing from the Rules are acceptable. If the provisions that depart from the Rules are contained in the parties' arbitration agreement, i.e. before the start of the proceedings, the Court may, if it considers the changes unacceptable, decide that the proceedings are not in fact ICC arbitration proceedings. In this case, it will refuse to administer the arbitration. These kinds of questions are sometimes unclear and, happily, they rarely arise in practice (in this regard, see our comments in relation to the arbitration agreement)» (Verbist et al., 2015, p. 77). 
mecanismo que, antes que dilatar el procedimiento arbitral, ayuda a las partes a ahorrar tiempo y dinero. Al respecto, el profesor Zahariev ha señalado expresamente lo siguiente: «It can also contribute to the timely resolution of the dispute, as it can prevent factual errors (typing errors, errors in calculations), or the omission of parts of the dispositive in the final version of the award. This will save time and money for the parties, as there would be no need to apply for the correction, supplementation or interpretation of the award» (2015).

Por todo lo expuesto, concluimos que las críticas al escrutinio de los laudos son absolutamente infundadas.

\section{EL ÉXITO DELESCRUTINIO DE LOS LAUDOS A NIVEL INTERNACIONAL}

Contrariamente a lo que se suele creer, no solo la CCI ha reconocido el escrutinio de los laudos. En efecto, una revisión de los reglamentos y de las prácticas de varias instituciones arbitrales refleja que no son pocas las instituciones arbitrales que realizan una revisión de los proyectos de laudo con respecto a los temas de forma ${ }^{23}$. Así, además de la CCI, destacan las siguientes instituciones arbitrales: China International Economic and Trade Arbitration Commission (CIETAC, artículo 51), Beijing Arbitration Commission (BAC); Singapore International Arbitration Centre (SIAC, artículo 28.2), International Commercial Arbitration Court at the Russian Federation Chamber of Commerce and Industry (ICAC, artículo 42), Court of Arbitration for Sport (CAS), Judicial Arbitration and Mediation Services (JAMS, artículo 32.3), Centro de Arbitraje de México (CAMEX, artículo 34.1); Kigali International Arbitration Centre (KIAC), Chambre Arbitrale Maritime (CAMP, artículo 27) and Danish Institution of Arbitration (DIA, artículo 28).

A manera de ejemplo, en relación con el escrutinio que se realiza en el SIAC, el profesor Born ha señalado expresamente lo siguiente:

The SIAC's organizational structure is similar to that of the ICC. It has a Court of Arbitration that comprises leading practitioners from the world over, and an experienced Secretariat with specialist lawyers

$23 \mathrm{Al}$ respecto, el profesor Gerbay ha señalado que «contrary to a commonly held belief, scrutiny is not a peculiarity found only in the ICC rules. A review of the rules and practices of many institutions indicate that the institutions that claim not to review at least the form of draft awards prior to their being issued to the parties are a minority. Such minority institutions include inter alia the CEPANI, the CRCICA, the BCICAC, the FCC (Helsinki), and the Arbitration Centre at the Chamber of Commerce of Caracas (Cámara de Caracas). On the other hand, a number of institutional rules, other than those of the ICC, contain express provisions on scrutiny. This is the case for instance of CIETAC, BAC, SIAC, ICAC, the CAS, J AMS, CAMEX, KIAC, CAMP and DIA. In addition, some other institutions review (more or less extensively) draft awards despite their rules being silent on the subject. This is the case, for example, of the ICDR, whose "staff review every award for completeness as to form". The same is true of the LCIA, the Swiss Institution, but also the NAI, the KCAB, the VIAC, the DIS » (2016, pp. 99-100).

NULLA EXECUTIO

SINE TITULO: EL

ESCRUTINIO DE

LOS LAUDOS EN

LA PRÁCTICA

DEL ARBITRAJE

INTERNACIONAL

COMO

MECANISMO

PARA OPTIMIZAR

LA EJECUCIÓN DE

LOS LAUDOS EN

EL PERÚ

NULLA EXECUTIO

SINE TITULO:THE

SCRUTINY OF

AWARDS IN THE

PRACTICE OF

INTERNATIONAL

ARBITRATION AS

A MECHANISM

TO OPTIMIZE THE

ENFORCEMENT

OF AWARDS IN

PERU 
to manage the administration of cases. Similar to the ICC, all SIAC awards are scrutinized in draft by the Registrar for issues of both form and substance, and can only be rendered by the tribunal if approved by the Registrar as to their form. While the Registrar manages the formal review and scrutiny process, the Registrar may, where appropriate, consult the SIAC Court before approving the draft award. According to the SIAC, timelines for scrutiny are shorter than at the ICC: two to three weeks for final awards, and one to two days in the case of emergency arbitrator orders and awards. The SIAC maintains a Panel of Arbitrators, which is composed of eminent international and regional arbitration practitioners who reflect a broad base of expertise, knowledge and experience (2015, p. 44).

Asimismo, existen otras instituciones que realizan un escrutinio del laudo a pesar de que su reglamento no lo prevé expresamente. Este es el caso, por ejemplo, del International Centre for Dispute Resolution (ICDR), cuyo staff revisa todos los laudos con respecto a la forma. Lo mismo sucede con las siguientes instituciones: London Court of International Arbitration (LCIA), Netherlands Arbitration Institute (NAI), Korean Commercial Arbitration Board (KCAB) y Vienna International Arbitral Centre (VIAC).

Si bien las recomendaciones de estas instituciones arbitrales no son formalmente vinculantes, lo cierto es que siempre son cumplidas por el tribunal arbitral sin mayores objeciones. Los tribunales arbitrales saben que las instituciones arbitrales tienen mucha experiencia con respecto a estas cuestiones, por lo que, a fin de evitar anulaciones y favorecer la ejecución del laudo, incorporan las recomendaciones brindadas por la institución sin reparos. Al respecto, el profesor Gerbay ha señalado expresamente que «an example is the informal scrutiny of draft awards which takes place at a number of institutions, despite the rules of these institutions being silent on the subject. For instance, this is the case of the Swiss Institution, the ICDR and the LCIA» (Gerbay, 2016, p. 107).

En nuestra opinión, el escrutinio informal que realizan estas instituciones arbitrales no vulnera la autonomía privada de las partes porque se trata de un mecanismo que es parte de la práctica del arbitraje internacional. En efecto, el escrutinio ha alcanzado tal nivel de aceptación a nivel internacional que difícilmente podría alegarse que se vulnera la autonomía privada de las partes. Precisamente este mecanismo, a pesar de no estar expresamente contemplado en un reglamento, satisface las expectativas y las necesidades de las partes.

Por otro lado, podemos encontrar otro ejemplo en el Reglamento del Stockholm Chamber of Commerce, en que se señala expresamente lo siguiente: «The SCC Rules does not have any service equivalent to the ICCs "scrutiny of award" but if the SCC Secretariat identifies any 
obvious miscalculations or similar it usually notifies the tribunal» (citado en Öhrström, 2013, p. 847).

También existen interesantes puntos medios. Por ejemplo, en la Corte de Arbitraje de la Cámara de Comercio e Industria de Bulgaria se contempla el escrutinio de los laudos, pero únicamente cuando los árbitros no son parte de la lista de árbitros de la institución arbitral. Sobre el particular, el profesor Gerbay ha señalado expresamente lo siguiente: "Interestingly, the rules of the Court of Arbitration at the Bulgarian Chamber of Commerce and Industry contemplate scrutiny, but only when the arbitrators are not on the panel of the institution. This "selective" scrutiny is to be conducted by a "committee of three arbitrators whose task is to check whether the award corresponds to the formal provisions of the Law on International Commercial Arbitration (LICA) and to the Rules of the Court of Arbitration. The findings of this committee are binding on the tribunal. See Article 37 of the rules of the Court of Arbitration at the Bulgarian Chamber of Commerce and Industry (1993)»(Gerbay, 2016, p. 107).

En atención a lo anterior, teniendo en cuenta el significativo número de instituciones arbitrales que han incorporado el escrutinio de los laudos, podemos aseverar con firmeza que se trata de un mecanismo que ha tenido mucho éxito en la práctica arbitral internacional.

\section{XIII.CONCLUSIONES}

1. La efectividad de los laudos en el ordenamiento jurídico peruano se ha visto relativizada, puesto que en múltiples casos los tribunales arbitrales no determinan con claridad los conceptos que integran las obligaciones contenidas en los laudos (costas, intereses, penalidades, etcétera). Esto ocasiona que, en reiteradas ocasiones, la parte vencida cuestione la ejecutabilidad del laudo $y$, en consecuencia, la efectividad del mismo se vea relativizada.

2. En los casos en los que los tribunales arbitrales no determinan con claridad los conceptos que integran las obligaciones contenidas en los laudos (costas, intereses, penalidades, etcétera), los jueces en ejecución no pueden liquidar lo que no es liquidable por falta de parámetros arbitrales. Los jueces se encuentran, así, en una situación trágica, puesto que, por un lado, emitir un pronunciamiento con respecto a dichos puntos implicaría violar una competencia ajena que le corresponde exclusivamente a los árbitros; mientras que, por otro lado, no pronunciarse con respecto a dichos puntos implicaría obligar a las partes a iniciar un nuevo arbitraje, asumir los costos del mismo y esperar años a que se dicte un nuevo laudo que se pronuncie sobre estos puntos.

NULLA EXECUTIO

SINE TITULO: EL

ESCRUTINIO DE

LOS LAUDOS EN

LA PRÁCTICA

DEL ARBITRAJE

INTERNACIONAL

COMO

MECANISMO

PARA OPTIMIZAR

LA EJECUCIÓN DE

LOS LAUDOS EN

EL PERÚ

NULLA EXECUTIO

SINE TITULO: THE

SCRUTINY OF

AWARDS IN THE

PRACTICE OF

INTERNATIONAL

ARBITRATION AS

A MECHANISM

TO OPTIMIZE THE

ENFORCEMENT

OF AWARDS IN

PERU 
3. Por un lado, si es que el juez en ejecución realizase una cognición sumaria para determinar las costas o los intereses, por ejemplo, estaría restringiendo la autonomía privada de los contratantes y la naturaleza del proceso de ejecución. Por otro lado, sin embargo, no determinar y luego ejecutar dichos conceptos implicaría restringir, entre otros derechos, la efectividad de las decisiones jurisdiccionales y la naturaleza del proceso en general, la cual implica resolver los conflictos de intereses.

4. Nos encontramos frente a un conflicto latente entre la autonomía privada y el derecho a la efectividad de las decisiones jurisdiccionales, donde el justiciable queda atrapado en el medio y sin solución pronta. Nuestra propuesta es el resultado de una ponderación de principios constitucionales. En nuestra opinión, al menos en el caso de los arbitrajes institucionales, el escrutinio de los laudos maximiza ambos principios constitucionales.

5. El escrutinio de los laudos es un mecanismo a través del cual un centro de arbitraje busca, a través de su reglamento, que el laudo sea de la mejor calidad posible, incrementando así las posibilidades de que sea ejecutado de forma efectiva. Asimismo, busca que el laudo esté debidamente motivado y con parámetros claros, de modo tal que el juez de ejecución pueda liquidar las obligaciones dinerarias bajo los parámetros de liquidación fijados por el tribunal arbitral, incrementando así las posibilidades de que el laudo sea cumplido voluntariamente por la parte perdedora ante la amenaza de una ejecución rápida y sin tropiezos. Por último, y como derivación de lo anterior, busca mitigar el riesgo de que el laudo sea anulado.

6. El escrutinio de los laudos salvaguarda la autonomía privada porque son las partes quienes voluntariamente deciden incorporar a su contrato un convenio arbitral sometiéndose a un reglamento arbitral que prevé el escrutinio de los laudos. En otras palabras, el escrutinio no es impuesto a las partes, sino que son estas quienes contractualmente deciden que la corte de arbitraje que administra el arbitraje intervenga antes de que se notifique el laudo a las partes, con el objetivo de mitigar el riesgo de anulación y garantizar la ejecutabilidad del laudo.

7. El escrutinio de los laudos salvaguarda también la efectividad de las decisiones jurisdiccionales, puesto que, precisamente, tiene como función incentivar el cumplimiento voluntario de la parte perdedora, garantizar la ejecutabilidad del laudo y mitigar el riesgo de que sea anulado.

8. Recomendamos a todos los centros de arbitraje peruanos que incorporen dentro de sus reglamentos arbitrales el escrutinio de 
los laudos. Así, podrían recoger en sus reglamentos de arbitraje una disposición muy similar a la que se encuentra prevista en el artículo 33 del reglamento de la $\mathrm{CCI}^{24}$. La siguiente regla modelo puede servir de inspiración para nuestros centros arbitrales:

Escrutinio de los laudos:

Antes de firmar un laudo, el tribunal arbitral deberá someterlo, en forma de proyecto, a la Corte. Esta podrá ordenar modificaciones de forma y, respetando la libertad de decisión del tribunal arbitral, podrá sugerirle precisiones sobre puntos relacionados con el fondo de la controversia, entre ellos, los parámetros a seguir por el juez al liquidar obligaciones dinerarias ordenadas por en el laudo. Al realizar el escrutinio de los laudos, la Corte no está ejerciendo una función jurisdiccional. Ningún laudo podrá ser dictado por el tribunal arbitral antes de haber sido aprobado por la Corte.

\section{REFERENCIAS}

Appel, M. (2012). International Centre for Dispute Resolution (ICDR). En K. Nairn \& P. Heneghan (eds.), Arbitration World: Institutional $\mathcal{E}$ Jurisdictional Comparisons (pp. 73-86). Londres: Thomson Reuters.

Ariano, E. (2010). La ejecución de garantías y algunos de sus (muchos) problemas. Cuadernos de investigación y jurisprudencia, 3(8), 85-94.

Blackaby, N., Partasides, C., Redfern, A. \& Hunter, M. (2015). Redfern and Hunter on international arbitration (6ta ed.). Alphen aan den Rijn: Kluwer Law International.

Bond, S. \& Paralika, M. (2015). ICC Rules of Arbitration, Awards, Article 33 [Scrutiny of the Award by the Court]. En L.A. Mistelis (ed.), Concise international arbitration (2da ed.). Alphen aan den Rijn: Kluwer Law International.

Born, G. B. (2015). International Arbitration: Law and Practice (2da ed.). Alphen aan den Rijn: Kluwer Law International.

Born, G. B. (2012). International commercial arbitration. Alphen aan den Rijn: Kluwer Law International.

Bustamante, R. (2013). La constitucionalización del arbitraje en el Perú: algunas consideraciones en torno a la relación del arbitraje con la Constitución, los derechos fundamentales y el Estado de Derecho. Derecho PUCP, 71, 387-411.

Carreteiro, M. A. (2016). Appellate arbitral rules in international commercial arbitration. Journal of International Arbitration, 33(2), 185-216.

MECANISMO

PARA OPTIMIZAR

LA EJECUCIÓN DE

LOS LAUDOS EN

EL PERÚ

NULLA EXECUTIO

SINE TITULO:THE

SCRUTINY OF

AWARDS IN THE

PRACTICE OF

INTERNATIONAL

ARBITRATION AS

A MECHANISM

TO OPTIMIZE THE

ENFORCEMENT

OF AWARDS IN

PERU 
Chamorro, F. (1994). La tutela judicial efectiva. Barcelona: Bosch.

Chen, F. (2007). Striving for independence, competence, and fairness: A case study of the Beijing Arbitration Commission. American Review of International Arbitration, 18, 313-352.

Cremades, B. (2006). El arbitraje en la doctrina constitucional española. Lima Arbitration, 1, 185-220.

De Oliveira, C. A. (2007). Del formalismo en el proceso civil. Lima: Palestra.

Feris, J. R. (2015). Presentación del Secretario General Adjunto de la CCI, en la sesión de la Academia de Arbitraje realizada en Paris el 1 de julio.

Fry, J. Greenberg S. and Mazza (2012). F. The Secretariat's Guiude to ICC Arbitration, ICC Publication, 729. Paris: International Court of Arbitration.

Gerbay, R. (2016). The functions of arbitral institutions. Alphen aan den Rijn: Kluwer Law International.

González, J. (2001). El derecho a la tutela jurisdiccional (3ra ed.). Madrid: Civitas.

Greenberg, S. (2013). Arbitral Award Scrutiny under Scrutiny. En P. Habegger, D. Hochstrasser, G. Nater-Bass \& U. Weber-Stecher (eds.), Arbitral Institutions Under Scrutiny (pp. 89-108). Nueva York: Juris Net.

Grigera, H. (1999). The powers of the ICC International Court of Arbitration vis-a-vis parties and arbitrators: Arbitration in the next decade. ICC Bulletin Special Supplement, 1999, 55-71.

International Chamber of Commerce (2009). 2008 Statistical Report. En ICC International Court of Arbitration Bulletin, 20(1).

International Chamber of Commerce (2010). 2009 Statistical Report. En ICC International Court of Arbitration Bulletin, 21(1).

International Chamber of Commerce (2011). 2010 Statistical Report. En ICC International Court of Arbitration Bulletin, 22(1).

International Chamber of Commerce (2012). 2011 Statistical Report. En ICC International Court of Arbitration Bulletin, 23(1).

International Chamber of Commerce (2013). 2012 Statistical Report. En ICC International Court of Arbitration Bulletin, 24 (1).

International Chamber of Commerce (2014). 2013 Statistical Report. En ICC International Court of Arbitration Bulletin, 25(1).

Leoveanu, A. L. (2016). Romanian Arbitration Law Issues in ICCParis Arbitration Practice. En L. Crenguta \& F. Baias (eds.), Arbitration in Romania: A Practitioner's Guide (pp. 336-352). Alphen aan den Rijn: Kluwer Law International.

Loquin, E. (1990). La sentence arbitrale: Lexamen du projet de sentence par l'institution et la sentence au deuxième degré - Réflexions sur la nature et la validité de l'intervention de l'institution arbitrale sur la sentence. Rev. Arbr., 427-464. 
Núñez del Prado, F. (2016). Desmitificando mitos: análisis económico de la doble instancia en el proceso civil peruano. Lima: Thomson Reuters.

Obando, V. R. (2011). Proceso civil y el derecho fundamental a la tutela jurisdiccional efectiva. Lima: Ara Editores.

Öhrström, M. (2013). The Arbitration Institute of the Stockholm Chamber of Commerce (SCC). En R. A. Schütze (ed.), Institutional Arbitration: Article-byArticle Commentary. Múnich: Beck.

Ortega, L. (2001). La ejecución de sentencias. En J. Leguina \& M. Sánchez (eds.), Comentarios a la Ley de la jurisdiccional contenciosa-administrativa (2da ed.). Valladolid: Lex Nova.

Palacios, E. (2009). Reflexiones sobre arbitraje y Constitución. En Constitución y proceso, Lima: Ara Editores.

Priori, G. (2003). La efectiva tutela jurisdiccional de las situaciones jurídicas materiales: hacia una necesaria reivindicación de los fines del proceso. Ius et Veritas, 26, 273-292.

Priori, G. (2009). Comentarios a la Ley del Proceso Contencioso Administrativo (4ta ed.). Lima: Ara Editores.

Rivarola, J. D. (2006). Comentario de la sentencia del Tribunal Constitucional: «El blindaje constitucional del arbitraje». Revista Peruana de Arbitraje, 2, 577-582.

Salcedo, C. M. (2008). La ejecución de la sentencia de tutela de derechos como derecho fundamental. Breves apuntes e implicancias a propósito del caso Fonavi. Actualidad Jurídica, 181, 201-206.

Verbist, H., Schäfer, E. \& Imhoos, C. (eds.). (2015). ICC Arbitration in Practice (2da ed.). Alphen aan den Rijn: Kluwer Law International.

Zahariev, Martin (2015). The scrutiny of an award: the Bulgarian arbitral institutions' perspective. Dimitrov, Petrov \& Co. Law Firm. Recuperado de http:// kluwerarbitrationblog.com/2015/10/29/the-scrutiny-of-an-award-the-bulgarianarbitral-institutions-perspective/

\section{Jurisprudencia, normativa y otros documentos legales}

Angeles García Barroso c. Juzgado de lo Social de Sevilla [recurso de amparo], Sentencia 163/1998, ECLI:ES:TC:1998:163 (Tribunal Constitucional [España], 14 de julio de 1998).

Código Procesal Civil [CPC] [Perú].

Colegiode Abogados deIcac. decretode urgencia [accióndeinconstitucionalidad], expedientes 015-2001-AI/TC y acumulados, sentencia (Tribunal Constitucional [Perú], 29 de enero de 2004). Consorcio Recupera Callao c. Municipalidad Provincial del Callao, expediente 06118-2010-90-1817-JR-CO-08, resolución 4 (Corte Superior de Justicia de Lima, Primera Sala Civil, Subespecialidad en Materia Comercial, 22 de noviembre de 2011).

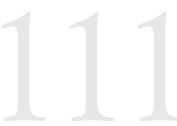

NULLA EXECUTIO SINE TITULO: EL ESCRUTINIO DE LOS LAUDOS EN LA PRÁCTICA DEL ARBITRAJE INTERNACIONAL COMO

MECANISMO

PARA OPTIMIZAR LA EJECUCIÓN DE LOS LAUDOS EN EL PERÚ

NULLA EXECUTIO SINE TITULO:THE SCRUTINY OF AWARDS IN THE PRACTICE OF INTERNATIONAL ARBITRATION AS A MECHANISM TO OPTIMIZE THE ENFORCEMENT OF AWARDS IN PERU 
Constitución Política del Perú (1993).

Coto Minero c. Audiencia Territorial de Valladolid [recurso de amparo], Sentencia 9/1981, ECLI:ES:TC:1981:9 (Tribunal Constitucional [España], 31 de marzo de 1981).

Fernando Cantuarias Salaverry c. Corte Superior de Justicia de Lima [hábeas corpus], expediente 6167-2005-PHC/TC, sentencia (Tribunal Constitucional [Perú], 28 de febrero de 2006)

Gerardo Fontán c. Audiencia Territorial de Madrid, Sentencia 28/1986, ECLI:ES:TC:1986:28 (Tribunal Constitucional [España], 20 de febrero 1986).

Gerardo Widauski c. Electrocentro, expediente 06578-2012-77-1817-JRCO-15, resolución (Corte Superior de Justicia de Lima, Segunda Sala Civil con Subespecialidad Comercial, 6 de junio de 2013).

Gerencia Comercial Inmobiliaria c. Electrocentro, expediente 05486-2011-801817-JR-CO-08, resolución 8 (Corte Superior de Justicia de Lima, Segunda Sala Civil con Subespecialidad Comercial, 6 de marzo de 2013).

Jaime Mamerto Ramírez Benavides c. Ministerio del Interior [acción de amparo], expediente 615-1999-AA/TC (Tribunal Constitucional [Perú], 26 de abril de 2000).

Julio Huamán Gómez c. Corporación José R. Lindley, expediente 01532-2011-0-1817-JR-CO-12, resolución 2 (Corte Superior de Justicia de Lima, Segunda Sala Civil con Subespecialidad Comercial, 11 de julio de 2012).

Ley de Arbitraje, Presidencia de la República, Decreto legislativo 1071, Diario Oficial El Peruano (28 de junio de 2008).

Ley Orgánica del Poder Judicial, Presidencia de la República [Perú], decreto supremo 017-93- JUS, Diario Oficial El Peruano (02 de junio de 1993). Tercera edición oficial del Texto Único Ordenado (2012), recuperado de https://www. minjus.gob.pe/wp-content/uploads/2014/09/DGDOJ-Ley-Orgánica-PoderJudicial-y- Ministerio-Público.pdf.

Livy Margot Chumacero Marticorena c. Gobierno Regional de Piura [acción de amparo], expediente 01797-2010-PA/TC (Tribunal Constitucional [Perú], 15 de noviembre de 2010).

María del Carmen Güell y otros c. Tribunal Superior de Justicia de Cataluña, Sentencia 298/1994, ECLI:ES:TC:1994:298 (Tribunal Constitucional [España], 14 de noviembre de 1994).

María Luisa Tárraga y otros c. Consejo Superior de Investigaciones Científicas, Sentencia 26/1983, ECLI:ES:TC:1983:26 (Tribunal Constitucinal [España], 13 de abril de 1983).

Mr X v Trioplast AB, Cour de Cassation, 1e civ., 11 de marzo de 2009, Bull. Civ. I, 48 (Francia). 
Société Cubic Defense Systems Inc. c. Chambre de Commerce Internationale, Cour d'Appel de Paris, 15 de septiembre de 1998, Rerue de l'Arbitrage, 1, 1999, p. 103 (Francia).

Société Cubic Defense Systems Inc. c. Chambre de Commerce Internationale, Tribunal de Grande Instance de Paris, 21 de mayo de 1997, Revue de l'Arbitrage, 3, 1997, p. 417 (Francia).

Société Cubic Defense Systems Inc. c. Chambre de Commerce Internationale, Cour de Cassation, 1e civ., 20 de febrero de 2001, Rerue de l'Arbitrage, 3, 2001, p. 511 (Francia).

Société Lear c. Chambre de Commerce Internationale, Tribunal de Grande Instance de Paris, 22 de mayo de 2002, Rerue de l'Arbitrage, 2002, p. 785 (Francia).

Société SNF SAS c Chambre de Commerce Internationale, Cour d'appel, 22 de junio de 2009, Revue de l'Arbitrage, 2010, p. 314 (Francia).

Société SNF SAS c Chambre de Commerce Internationale, Tribunal de Grande Instance de Paris, 10 de octubre de 2007, Revue de l'Arbitrage, 4, 2007, p. 847 (Francia).

Suring SAC c. Ministerio de Educación, expediente 03383-2011-27-1817-JRCO-17, resolución 2 (Corte Superior de Justicia de Lima, Segunda Sala Civil con Subespecialidad Comercial, 05 de junio de 2002).

NULLA EXECUTIO SINE TITULO: EL ESCRUTINIO DE

LOS LAUDOS EN

LA PRÁCTICA

DEL ARBITRAJE

INTERNACIONAL COMO

MECANISMO

PARA OPTIMIZAR LA EJECUCIÓN DE LOS LAUDOS EN

EL PERÚ

NULLA EXECUTIO

SINE TITULO: THE

SCRUTINY OF

AWARDS IN THE

PRACTICE OF

INTERNATIONAL

ARBITRATION AS

A MECHANISM

TO OPTIMIZE THE

ENFORCEMENT

OF AWARDS IN

PERU 